\title{
ON PROPER HARMONIC MAPS BETWEEN STRICTLY PSEUDOCONVEX DOMAINS WITH KÄHLER METRICS OF BERGMAN TYPE*
}

\author{
SONG-YING $\mathrm{LI}^{\dagger}$ AND EZEQUIAS SIMON ${ }^{\ddagger}$
}

Dedicated to Salah Baouendi for his seventieth birthday

Key words. Proper harmonic maps, pluriharmonic maps and holomorphic maps, rigidity

AMS subject classifications. 32W50, 35B65

1. Introduction. Let $M$ and $N$ be Kähler manifolds with respective Kähler metrics $h=h_{i \bar{j}} d z_{i} \otimes d \bar{z}_{j}$ and $g=g_{\alpha \bar{\beta}} d w^{\alpha} \otimes d \bar{w}^{\beta}$, respectively. A map $u: M^{m} \rightarrow N^{n}$ is said to be harmonic if the tension field $\tau^{s}[u]$ satisfies

$$
\tau^{s}[u]=\Delta_{M} u^{s}+\sum_{i, j=1}^{m} \sum_{t, \gamma=1}^{n} \Gamma_{t \gamma}^{s} \partial_{i} u^{t} \partial_{\bar{j}} u^{\gamma} h^{i \bar{j}}=0 \text { for } 1 \leq s \leq n,
$$

where $\left(h^{i \bar{j}}\right)^{t}$ is the inverse of the matrix $\left(h_{i \bar{j}}\right), \Delta_{M}=\sum_{i, j} h^{i \bar{j}} \partial_{i \bar{j}}$ and $\Gamma_{t \gamma}^{s}$ denote the Christoffel symbols of the Hermitian metric $g$ on $N$. It follows from (1.1) that if $u$ is holomorphic, then $u$ must be harmonic. Thus, it is natural to ask under what circumstances a harmonic map is holomorphic or antiholomorphic. Under the assumption that both $M$ and $N$ are compact, Siu [31] demonstrated that if the curvature tensor of $N$ is strongly negative and the rank of $d u$ is greater than or equal to four at a point of $M$, then a harmonic map $u$ must be holomorphic or antiholomorphic. The proof follows from Siu's Bochner type identity together with the compactness assumption on $M$.

If $M$ is a complete noncompact manifold of strongly negative curvature with infinite volume, the previous Bochner type identity technique fails and not much is known about the rigidity of $u$. In general, the answer to the above posed question is negative: one needs to add some natural conditions to the map such as being a proper map. Along this direction, when $M$ and $N$ are unit balls in $\mathbb{C}^{n}$ endowed with Bergman metrics (the simplest case of Kähler manifolds with strongly negative curvature) progress was made by $\mathrm{Li}$ and $\mathrm{Ni}$ in [25]. They showed that for $m>1$, if $u:\left(B^{m}, h\right) \rightarrow\left(B^{n}, g\right)$ is a $C^{2}$ up to the boundary pluriharmonic proper map, where $h$ and $g$ are respective Bergman metrics on $B^{m}$ and $B^{n}$, then $u$ must be holomorphic or antiholomorphic. In addition to this, several other equivalent conditions were given (cf. [25]).

The main purpose of this paper is to use a similar approach to the one given in (cf. [25]) to generalize their theorem from unit balls to smoothly bounded strictly pseudoconvex domains in $\mathbb{C}^{m}$ and $\mathbb{C}^{n}$ for $m>1$ with more general metrics of Bergman type. More precisely, we consider two smoothly bounded strictly pseudoconvex domains $\Omega_{m}$ and $\Omega_{n}$ in $\mathbb{C}^{m}$ and $\mathbb{C}^{n}$ respectively. Let $\rho$ and $r$ be $C^{4}$ respective strictly

\footnotetext{
${ }^{*}$ Received March 23, 2006; accepted for publication August 31, 2006.

$\dagger$ Department of Mathematics, University of California, Irvine, CA 92697, USA (sli@math.uci.edu).

${ }^{\ddagger}$ Department of Mathematics, University of California, Santa Cruz, CA 95046, USA (simon@ math.ucsc.edu).
} 
plurisubharmonic defining functions for $\Omega_{m}$ and $\Omega_{n}$. We consider the complex Kähler metric

$$
h=h_{i \bar{j}} d z_{i} \otimes d \bar{z}_{j}=-\frac{\partial^{2} \log (-\rho)}{\partial z_{i} \otimes \partial \bar{z}_{j}} d z_{i} \otimes d \bar{z}_{j}
$$

for $\Omega_{m}$ and the Kähler metric

$$
g=g_{\alpha \bar{\beta}} d w^{\alpha} \otimes d \bar{w}^{\beta}=-\frac{\partial^{2} \log (-r)}{\partial w^{\alpha} \partial \bar{w}^{\beta}} d w^{\alpha} \otimes d \bar{w}^{\beta}
$$

for $\Omega_{n}$. By the asymptotic expansion of the Bergman kernel function given by C. Fefferman in [15] the Bergman metric is a special case of the above setting, and so is the Kähler-Einstein metric given by Cheng and Yau in [8].

Let $\left(\rho^{i \bar{j}}\right)^{t}$ be the inverse matrix of the matrix $\left(\rho_{i \bar{j}}\right)$. Let

$$
\rho^{i}=\sum_{j} \rho^{i \bar{j}} \rho_{\bar{j}}, \quad \rho^{\bar{j}}=\sum_{i} \rho^{i \bar{j}} \rho_{i} \text { and }|\partial \rho|_{\rho}^{2}=\sum_{i} \rho^{i} \rho_{i}=\sum_{j} \rho^{\bar{j}} \rho_{\bar{j}} ;
$$

a complex normal derivative $R$, a tangential complex derivative $X_{j}$ and an elliptic operator $\mathcal{L}$ be defined as follows:

$$
R:=\rho^{j} \partial_{j}, \quad X_{j}:=\sum_{i=1}^{m}\left(\rho^{i \bar{j}}-\frac{\rho^{i} \rho^{\bar{j}}}{|\partial \rho|_{\rho}^{2}-\rho}\right) \partial_{i}, \quad \mathcal{L}:=\left(\rho^{i \bar{j}}-\frac{\rho^{i} \rho^{\bar{j}}}{|\partial \rho|_{\rho}^{2}-\rho}\right) \partial_{i \bar{j}} .
$$

Let $e[u]$ be the energy density function associated to the map $u:(M, h) \rightarrow(N, g)$ defined as

$$
e[u](z):=\sum_{\alpha, \beta=1}^{n} \sum_{i, j=1}^{m} h^{i \bar{j}} g_{\alpha \bar{\beta}}\left(\partial_{i} u^{\alpha} \overline{\partial_{j} u^{\beta}}+\partial_{\bar{j}} u^{\alpha} \overline{\partial_{\bar{i}} u^{\beta}}\right)
$$

Our first theorem, which is a generalization of the main theorem for the case when $\Omega_{m}=B_{m}$ and $\Omega_{n}=B_{n}$ are balls given by $\mathrm{Li}$ and $\mathrm{Ni}$ in [25], is as follows:

Theorem 1.1. Assume that $m>1$, and let $\Omega_{m} \subset \mathbb{C}^{m}, \Omega_{n} \subset \mathbb{C}^{n}$ be bounded strictly pseudoconvex domains with strictly plurisubharmonic defining functions $\rho \in$ $C^{4}\left(\bar{\Omega}_{m}\right), r \in C^{4}\left(\bar{\Omega}_{n}\right)$, respectively. Let $u: \Omega_{m} \rightarrow \Omega_{n}$ be a proper map so that $u \in$ $C^{2}\left(\bar{\Omega}_{m}, \bar{\Omega}_{n}\right)$. Then the following statements are equivalent.

(i) The map $u$ is either holomorphic or antiholomorphic.

(ii) The map u is pluriharmonic.

(iii) The map $u$ is harmonic and $r_{s} \mathcal{L} u^{s}+r_{s t} X_{j} u^{t} u \frac{s}{j}=0$ on $\partial \Omega_{m}$.

(iv) The map $u$ is harmonic and the energy density function $e[u](z)=m$ on the set $\left\{z \in \partial \Omega_{m}: E_{b}[u](z)>0\right\}$, where $E_{b}[u](z)=\left|\bar{\partial}_{b} u\right|^{2}+\left|\partial_{b} u\right|^{2}$ and $\bar{\partial}_{b}$ is the tangential Cauchy-Riemann operator.

(v) The map $u$ is harmonic and $\sum_{\gamma, s=1}^{n}\left(r_{s} R u^{s}(z)\right)\left(r_{\gamma} \bar{R} u^{\gamma}(z)\right)=0$ on $\partial \Omega_{m}$.

Another problem we want to explore is the existence and regularity of proper harmonic maps. More precisely, if $\phi: \partial \Omega_{m} \rightarrow \partial \Omega_{n}$ is a smooth map, can one find a harmonic map $u$ that when restricted to $\partial \Omega_{m}$ equals $\phi$ ? If so, what type of regularity statement can we offer?

In a series of papers [19]-[21], P. Li and L-F. Tam explored the existence, uniqueness and regularity of proper harmonic maps between real hyperbolic spaces. They 
showed that if $\phi: D^{m} \rightarrow D^{n}$ (here $D^{m}$ is the unit ball in $\mathbb{R}^{m}$ with the Poincaré metric and similarly $D^{n}$ is the unit ball in $\mathbb{R}^{n}$ with the Poincare metric) is a $C^{1}$ map with nonvanishing energy density $e(\phi)(x)$ at every $x \in S^{m-1}$, then there exists a unique proper harmonic map $u: D^{m} \rightarrow D^{n}$ which equals $\phi$ when restricted to $S^{m-1}$. If in addition, the boundary map $\phi$ is in $C^{k, \alpha}\left(S^{m-1}, S^{n-1}\right)$, where $1 \leq k \leq m-1$ and $0<\alpha \leq 1$, then $u$ belongs to $C^{k, \gamma}\left(\bar{D}^{m}\right)$ for $0<\gamma<\alpha$. They also proved that if $u: \bar{D}^{m} \rightarrow \bar{D}^{n}$ and $v: \bar{D}^{m} \rightarrow \bar{D}^{n}$ are $C^{1}$ proper harmonic maps such that they are equal on $S^{m-1}$ and the energy density of the boundary map does not vanish anywhere, then $u=v$. As a corollary, they obtained that if $u: \bar{D}^{m} \rightarrow \bar{D}^{n}$ is a $C^{1}$, proper harmonic map with non-vanishing energy density on $S^{m-1}$, then the energy density equals $m$ at the boundary.

The case where both $M$ and $N$ are rank one symmetric spaces was tackled by Donnelly in [11]. He was able to generalize the existence and regularity results of $\mathrm{Li}$ and Tam under the assumption that the boundary map $\phi$ satisfies some contact conditions. The problem was also studied by S-Y. Li and L. Ni in [25] where they formulated a simpler contact condition and provided an existence theorem. The second purpose of this paper is to generalize their theorem on unit balls to strictly pseudoconvex domains. Our result is:

Theorem 1.2. Assume that $\phi: \partial \Omega_{m} \rightarrow \partial \Omega_{n}$ belongs to $C^{k, \alpha}\left(\partial B_{m}\right)$ for $k \geq 2$ and $0<\alpha \leq 1$. In addition, suppose that $E_{b}[\phi](z)=\left|\bar{\partial}_{b} \phi\right|^{2}+\left|\partial_{b} \phi\right|^{2}>0$ on $\partial \Omega_{m}$, where $\bar{\partial}_{b}$ is the tangential Cauchy-Riemann operator, and the necessary condition

$$
\sum_{s} r_{s}(\phi)(z) X_{j} \phi^{s}(z)=0, \quad z \in \partial \Omega_{m}, \quad 1 \leq j \leq m
$$

Then for all $0<l+\beta<\min \{m, k+\alpha\}$ there exists a unique proper harmonic map $u \in C^{l, \beta}\left(\bar{\Omega}_{m}\right)$ such that $u=\phi$ on $\partial \Omega_{m}$.

2. Cauchy-Riemann functions. A complex-valued $C^{1}$ function $u$ in a domain $\Omega$ in $\mathbb{C}^{m}$ is said to be CR if $\bar{\partial} u=0$, which is the same as $u$ being holomorphic. Since $\frac{\partial}{\partial z_{j}} \frac{\partial}{\partial \bar{z}_{k}}=\frac{\partial}{\partial \bar{z}_{k}} \frac{\partial}{\partial z_{j}}$ for all $1 \leq j, k \leq m$, it is easy to show that if for each $z \in \Omega$ we have that either $\bar{\partial} u(z)=0$ or $\bar{\partial} \bar{u}(z)=0$, then we must have that either $\bar{\partial} u \equiv 0$ on $\Omega$ or $\bar{\partial} \bar{u} \equiv 0$ on $\Omega$.

It was proved by $\mathrm{Li}$ and $\mathrm{Ni}[25]$ that the above phenomenon remains true for functions on the unit sphere in $\mathbb{C}^{m}(m>1)$ where the problem is much more difficult since the tangent vector fields are not commutative.

Let $\Omega$ be a smoothly bounded domain in $\mathbb{C}^{m}$. Let $u \in C^{1}(\partial \Omega)$. We say that $u$ is a CR function on $\partial \Omega$ if $u$ satisfies the tangential Cauchy-Riemann equation: $\bar{\partial}_{b} u=0$ on $\partial \Omega$, which is equivalent to $\bar{X}_{j} u=0$ on $\partial \Omega$ for all $1 \leq j \leq m$ where $X_{1}, \cdots, X_{m}$ are holomorphic tangent vector fields which span the holomorphic tangent bundle on $\partial \Omega$. Based on the main idea in [25], Li and Zhang [27] proved the following theorem.

TheOREM 2.1. Let $m>1$ and let $\Omega$ be a bounded strictly pseudoconvex domain in $\mathbb{C}^{m}$ with $C^{3}$ boundary. Let $g \in C^{2}(\partial \Omega)$ so that for any point $z \in \partial \Omega$, we have that either $\bar{\partial}_{b} g(z)=0$ or $\bar{\partial}_{b} \bar{g}(z)=0$. Then either $g$ is $C R$ or $\bar{g}$ is $C R$ on $\partial \Omega$.

3. Preliminary results. Let $u=\left(u^{1}, u^{2}, \ldots, u^{n}\right):(M, h) \rightarrow(N, g)$ be a map. We need the following definitions: 
(i) $u$ is pluriharmonic if

$$
\partial_{i \bar{j}} u^{s}+\sum_{t, \gamma=1}^{n} \Gamma_{t \gamma}^{s} \partial_{i} u^{t} \partial_{\bar{j}} u^{\gamma}=0 \text { for } 1 \leq i, j \leq m \text { and } 1 \leq s \leq n .
$$

(ii) $u$ is holomorphic if $\partial_{\bar{i}} u^{s}=0$ for $1 \leq i \leq m$ and $1 \leq s \leq n$.

Since

(iii) the energy density function of $u$ denoted $e[u](z)$ is defined by (1.6).

$$
h_{i \bar{j}}=-\frac{\partial}{\partial z_{i}}\left(\frac{\rho_{\bar{j}}}{\rho}\right)=\frac{\rho_{i \bar{j}}}{-\rho}+\frac{\rho_{i} \rho_{\bar{j}}}{\rho^{2}}=\frac{1}{-\rho}\left[\rho_{i \bar{j}}+\frac{\rho_{i} \rho_{\bar{j}}}{-\rho}\right],
$$

we have

$$
h^{i \bar{j}}=(-\rho)\left[\rho^{i \bar{j}}-\frac{\rho^{i} \rho^{\bar{j}}}{|\partial \rho|_{\rho}^{2}-\rho}\right]
$$

where

$$
\rho^{i}=\sum_{j} \rho^{i \bar{j}} \rho_{\bar{j}}, \rho^{\bar{j}}=\sum_{i} \rho^{i \bar{j}} \rho_{i} \text { and }|\partial \rho|_{\rho}^{2}=\sum_{i} \rho^{i} \rho_{i}=\sum_{j} \rho^{\bar{j}} \rho_{\bar{j}} .
$$

Therefore

$$
r_{i \bar{l}} r^{\bar{l}}=r_{i \bar{l}} r^{p \bar{l}} r_{p}=\delta_{i p} r_{p}=r_{i}, \quad r_{j \bar{l}} r^{\bar{l}}=r_{j \bar{l}} r^{p \bar{l}} r_{p}=\delta_{j p} r_{p}=r_{j} .
$$

Let $\mathcal{L}, R$ and $X_{j}$ be defined by (1.5). Then

$$
\bar{X}_{i}=\sum_{j=1}^{m}\left(\rho^{i \bar{j}}-\frac{\rho^{i} \rho^{\bar{j}}}{|\partial \rho|_{\rho}^{2}-\rho}\right) \partial_{\bar{j}} \quad \text { and } \quad \bar{R}=\sum_{j=1}^{m} \rho^{\bar{j}} \partial_{\bar{j}} .
$$

For $u \in C^{2}\left(\bar{\Omega}_{m}, \bar{\Omega}_{n}\right)$, let

$$
E_{b}(u):=\left|\partial_{b} u\right|^{2}+\left|\bar{\partial}_{b} u\right|^{2}
$$

where

$$
\left|\partial_{b} u\right|^{2}=\sum_{j=1}^{m} \sum_{s=1}^{n}\left|X_{j} u^{s}\right|^{2},\left|\bar{\partial}_{b} u\right|^{2}=\sum_{j=1}^{m} \sum_{s=1}^{n}\left|\bar{X}_{j} u^{s}\right|^{2} .
$$

Finally, since $\rho(z)<0$ on $\Omega_{m}$, let

$$
a[u](z)=\frac{r(u(z))}{\rho(z)}, \quad z \in \Omega_{m} .
$$

For each $z_{0} \in \partial \Omega_{m}, a[u]\left(z_{0}\right)=\lim _{z \rightarrow z_{0}} a[u](z)$. We will apply this convention for the rest of the paper.

Now we proceed to compute $\tau^{s}[u]$ explicitly. Then using the properness assumption on $u$, we obtain an expression for $\tau^{s}[u]$ that allows us to understand under what circumstances either $u$ is $\mathrm{CR}$ or $\bar{u}$ is CR.

Let us first obtain an explicit expression for the Christoffel symbols $\Gamma_{i j}^{k}$ for $N=$ $\left(\Omega_{n}, g\right)$. By definition we know that 


$$
\begin{aligned}
& \Gamma_{i j}^{k}=\sum_{\ell} g^{k \bar{\ell}} \frac{\partial g_{i \bar{\ell}}}{\partial u^{j}} \\
& =(-r)\left(r^{k \bar{\ell}}-\frac{r^{k} r^{\bar{\ell}}}{|\partial r|_{r}^{2}-r}\right) \frac{\partial}{\partial u^{j}}\left[\frac{1}{(-r)}\left(r_{i \bar{\ell}}+\frac{r_{i} r_{\bar{\ell}}}{-r}\right)\right] \\
& =(-r)\left(r^{k \bar{\ell}}-\frac{r^{k} r^{\bar{\ell}}}{|\partial r|_{r}^{2}-r}\right)\left[\frac{r_{j}}{r^{2}}\left(r_{i \bar{\ell}}+\frac{r_{i} r_{\bar{\ell}}}{-r}\right)+\frac{1}{(-r)}\left(r_{i j \bar{\ell}}+\frac{r_{j}}{r^{2}} r_{i} r_{\bar{\ell}}+\frac{r_{i j} r_{\bar{\ell}}+r_{i} r_{j \bar{\ell}}}{-r}\right)\right] \\
& =\left(r^{k \bar{\ell}}-\frac{r^{k} r^{\bar{\ell}}}{|\partial r|_{r}^{2}-r}\right)\left[\frac{r_{j}}{-r}\left(r_{i \bar{\ell}}+\frac{r_{i} r_{\bar{\ell}}}{-r}\right)+r_{i j \bar{\ell}}+\frac{r_{j}}{r^{2}} r_{i} r_{\bar{\ell}}+\frac{r_{i j} r_{\bar{\ell}}+r_{i} r_{j \bar{\ell}}}{-r}\right] \\
& =\left(r^{k \bar{\ell}}-\frac{r^{k} r^{\bar{\ell}}}{|\partial r|_{r}^{2}-r}\right)\left[r_{i j \bar{\ell}}+\frac{2}{r^{2}} r_{i} r_{j} r_{\bar{\ell}}+\frac{r_{i \bar{\ell}} r_{j}+r_{i j} r_{\bar{\ell}}+r_{i} r_{j \bar{\ell}}}{-r}\right] \\
& =\left(r^{k \bar{\ell}}-\frac{r^{k} r^{\bar{\ell}}}{\left|\partial r_{r}\right|^{2}-r}\right) r_{i j \bar{\ell}}+\left(r^{k \bar{\ell}}-\frac{r^{k} r^{\bar{\ell}}}{|\partial r|_{r}^{2}-r}\right)\left(\frac{r_{i \bar{\ell}} r_{j}+r_{i j} r_{\bar{\ell}}+r_{i} r_{j \bar{\ell}}}{-r}\right) \\
& +\frac{2}{r^{2}}\left(r_{i} r_{j} r^{k}-\frac{|\partial r|_{r}^{2}}{|\partial r|_{r}^{2}-r} r^{k} r_{i} r_{j}\right) \\
& =\left(r^{k \bar{\ell}}-\frac{r^{k} r^{\bar{\ell}}}{|\partial r|_{r}^{2}-r}\right) r_{i j \bar{\ell}}+\frac{1}{(-r)}\left(\delta_{i k} r_{j}+r^{k} r_{i j}+r_{i} \delta_{j k}\right) \\
& -\frac{r^{k} r^{\bar{\ell}}}{(-r)\left(|\partial r|_{r}^{2}-r\right)}\left(r_{i \bar{\ell}} r_{j}+r_{i j} r_{\bar{\ell}}+r_{i} r_{j \bar{\ell}}\right)+\frac{2}{(-r)} \frac{r^{k} r_{i} r_{j}}{\left(|\partial r|_{r}^{2}-r\right)} \\
& =\left(r^{k \bar{\ell}}-\frac{r^{k} r^{\bar{\ell}}}{|\partial r|_{r}^{2}-r}\right) r_{i j \bar{\ell}}+\frac{1}{(-r)}\left(\delta_{i k} r_{j}+r^{k} r_{i j}+r_{i} \delta_{j k}\right) \\
& -\frac{1}{(-r)\left(|\partial r|_{r}^{2}-r\right)}\left(r_{i} r^{k} r_{j}+r_{i j} r^{k}|\partial r|_{r}^{2}+r_{i} r^{k} r_{j}\right)+\frac{2}{(-r)} \frac{r^{k} r_{i} r_{j}}{\left(|\partial r|_{r}^{2}-r\right)} \\
& =\left(r^{k \bar{\ell}}-\frac{r^{k} r^{\bar{\ell}}}{|\partial r|_{r}^{2}-r}\right) r_{i j \bar{\ell}}+\frac{1}{(-r)}\left(\delta_{i k} r_{j}+r^{k} r_{i j}+r_{i} \delta_{j k}\right) \\
& -\frac{|\partial r|_{r}^{2}}{(-r)\left(|\partial r|_{r}^{2}-r\right)} r_{i j} r^{k} \\
& =\frac{1}{-r}\left(\delta_{i k} r_{j}+r_{i} \delta_{j k}\right)+\left(r^{k \bar{\ell}}-\frac{r^{k} r^{\bar{\ell}}}{|\partial r|_{r}^{2}-r}\right) r_{i j \bar{\ell}}+\frac{r_{i j} r^{k}}{|\partial r|_{r}^{2}-r} \text {. }
\end{aligned}
$$

Substituting the expression we just found for $\Gamma_{i j}^{k}$ in the definition of $\tau^{s}[u]$, we obtain

$$
\begin{aligned}
\tau^{s}[u]= & \Delta_{M} u^{s}+\frac{1}{(-r(u))} h^{i \bar{j}}\left(\delta_{t s} r_{\gamma}(u)+r_{t}(u) \delta_{\gamma s}\right) u_{i}^{t} u \frac{\gamma}{j} \\
& +\left[\left(r^{s \bar{\ell}}(u)-\frac{r^{s}(u) r^{\bar{\ell}}(u)}{|\partial r|_{r}^{2}(u)-r(u)}\right) r_{t \gamma \bar{\ell}}(u)+\frac{r_{t \gamma}(u) r^{s}(u)}{\left(|\partial r|_{r}^{2}-r(u)\right)}\right] h^{i \bar{j}} u_{i}^{t} u \frac{\gamma}{j} \\
= & \Delta_{M} u^{s}+\frac{1}{(-r(u))} h^{i \bar{j}}\left(r_{\gamma}(u) u_{i}^{s} u \frac{\gamma}{j}+r_{t}(u) u_{i}^{t} u \frac{s}{j}\right) \\
& +\left[\left(r^{s \bar{\ell}}(u)-\frac{r^{s} r^{\bar{\ell}}(u)}{|\partial r|_{r}^{2}-r(u)}\right) r_{t \gamma \bar{\ell}}(u)+\frac{r_{t \gamma}(u) r^{s}(u)}{|\partial r|_{r}^{2}-r(u)}\right] h^{i \bar{j}} u_{i}^{t} u \frac{\gamma}{j} .
\end{aligned}
$$


Let $u=\left(u_{1}, \cdots, u_{n}\right) \in C^{2}\left(\bar{\Omega}_{m}\right)$ be a map from $\Omega_{m} \rightarrow \Omega_{n}$. Define

$$
E^{s}[u]:=\frac{1}{(-\rho)} h^{i \bar{j}}\left[r_{\gamma}(u) u_{i}^{s} u \frac{\gamma}{j}+r_{t}(u) u_{i}^{t} u \frac{s}{j}\right] .
$$

Then using the expression for $h^{i \bar{j}}$ in (3.3), we have

$$
\begin{aligned}
E^{s}[u] & =\left(\rho^{i \bar{j}}-\frac{\rho^{i} \rho^{\bar{j}}}{|\partial \rho|_{\rho}^{2}-\rho}\right)\left[r_{t}(u) u_{i}^{s} \bar{\partial}_{j} u^{t}+r_{t}(u) \partial_{i} u^{t} u \frac{s}{j}\right] \\
& =u_{i}^{s} r_{t}(u) \bar{X}_{i} u^{t}+u \frac{s}{i} r_{t}(u) X_{i} u^{t} .
\end{aligned}
$$

Next we will express $E^{s}[u]$ in terms of the vector fields $X_{j}, R, \bar{X}_{j}$ and $\bar{R}$. This is carried out in the following lemma.

Lemma 3.1. Let $u=\left(u^{1}, \cdots, u^{n}\right): \Omega_{m} \rightarrow \Omega_{n}$ be a map with $u \in C^{2}\left(\bar{\Omega}_{m}\right)$. Then

$$
\begin{aligned}
E^{s}[u]= & \rho_{i \bar{j}}\left[X_{i} u^{s} r_{t}(u) \bar{X}_{j} u^{t}+\bar{X}_{j} u^{s} r_{t}(u) X_{i} u^{t}\right] \\
& +\frac{-\rho}{\left(|\partial \rho|_{\rho}^{2}-\rho\right)^{2}}\left[R u^{s} r_{t}(u) \bar{R} u^{t}+\bar{R} u^{s} r_{t}(u) R u^{t}\right]
\end{aligned}
$$

for all $z \in \bar{\Omega}_{m}$.

Proof. For any point $z_{0} \in \bar{\Omega}_{m}$, by a rotation if necessary, we may assume that the complex Hessian matrix of $\rho$ at $z_{0}$ is diagonal. In other words, we may assume that $H(\rho)\left(z_{0}\right)=\operatorname{diag}\left(\rho_{1 \overline{1}}, \cdots, \rho_{m \bar{m}}\right)$. Thus $\rho^{i \bar{j}}\left(z_{0}\right)=\delta_{i j} \rho_{i \bar{i}}\left(z_{0}\right)^{-1}$ and

$$
\begin{aligned}
E^{s}[u]= & u_{i}^{s} r_{t}(u) \bar{X}_{i} u^{t}+u \frac{s}{i} r_{t}(u) X_{i} u^{t} \\
= & \rho_{i \bar{i}} X_{i} u^{s} r_{t}(u) \bar{X}_{i} u^{t}+\rho_{i \bar{i}} \frac{\rho^{\ell} \rho^{\bar{i}}}{|\partial \rho|_{\rho}^{2}-\rho} u_{\ell}^{s} r_{t}(u) \bar{X}_{i} u^{t} \\
& +\rho_{i \bar{i}} \bar{X}_{i} u^{s} r_{t}(u) X_{i} u^{t}+\rho_{i \bar{i}} \frac{\rho^{i} \rho^{\bar{\ell}}}{|\partial \rho|_{\rho}^{2}-\rho} u_{\bar{\ell}}^{s} r_{t}(u) X_{i} u^{t} \\
= & \rho_{i \bar{i}}\left[X_{i} u^{s} r_{t}(u) \bar{X}_{i} u^{t}+\bar{X}_{i} u^{s} r_{t}(u) X_{i} u^{t}\right] \\
& +\rho_{i \bar{i}}\left[\frac{\rho^{\bar{i}}}{|\partial \rho|_{\rho}^{2}-\rho} R u^{s} r_{t}(u) \bar{X}_{i} u^{t}+\frac{\rho^{i}}{|\partial \rho|_{\rho}^{2}-\rho} \bar{R} u^{s} r_{t}(u) X_{i} u^{t}\right] .
\end{aligned}
$$

Note that at $z=z_{0}$, we have that $\rho_{i \bar{i}} \rho^{\bar{i}}=\rho_{i}$ and $\rho_{i} \rho^{i \bar{i}}=\rho^{\bar{i}}$. Thus

$$
\begin{aligned}
\sum_{i=1}^{m} \rho_{i \bar{i}} \rho^{\bar{i}} \bar{X}_{i}=\sum_{i=1}^{m} \rho_{i} \bar{X}_{i} & =\sum_{i=1}^{m} \rho_{i} \sum_{j=1}^{m}\left(\rho^{i \bar{j}}-\frac{\rho^{i} \rho^{\bar{j}}}{|\partial \rho|_{\rho}^{2}-\rho}\right) \partial_{\bar{j}} \\
& =\sum_{i=1}^{m} \rho^{\bar{i}} \partial_{\bar{i}}-\sum_{j=1}^{m} \frac{|\partial \rho|_{\rho}^{2} \rho^{\bar{j}}}{|\partial \rho|_{\rho}^{2}-\rho} \partial_{\bar{j}} \\
& =\bar{R}-\frac{|\partial \rho|_{\rho}^{2} \bar{R}}{|\partial \rho|_{\rho}^{2}-\rho} \\
& =\frac{-\rho}{|\partial \rho|_{\rho}^{2}-\rho} \bar{R} .
\end{aligned}
$$


Therefore,

$$
\begin{aligned}
E^{s}[u]= & \rho_{i \bar{i}}\left[X_{i} u^{s} r_{t}(u) \bar{X}_{i} u^{t}+\bar{X}_{i} u^{s} r_{t}(u) X_{i} u^{t}\right] \\
& +\frac{-\rho}{\left(|\partial \rho|_{\rho}^{2}-\rho\right)^{2}}\left[R u^{s} r_{t}(u) \bar{R} u^{t}+\bar{R} u^{s} r_{t}(u) R u^{t}\right] .
\end{aligned}
$$

The proof is complete. $\quad \square$

Next, we want to understand the behavior of $r_{s} X_{j} u^{s}$ and $r_{s} \bar{X}_{j} u^{s}$ on $\partial \Omega_{m}$. This is the content of Lemma 3.2.

LEMmA 3.2. Let $u=\left(u_{1}, \cdots, u_{n}\right): \Omega_{m} \rightarrow \Omega_{n}$ be a proper harmonic map so that $u \in C^{2}\left(\bar{\Omega}_{m}\right)$. Then

$$
\sum_{s=1}^{n} r_{s}(u) \bar{\partial}_{b} u^{s}=\sum_{s=1}^{n} r_{s}(u) \partial_{b} u^{s} \equiv 0 \quad \text { on } \quad \partial \Omega_{m} .
$$

Proof. By Lemma 3.1

$$
r_{s}(u) E^{s}[u]=2 \rho_{i \bar{j}} r_{s}(u) X_{i} u^{s} r_{t}(u) \bar{X}_{j} u^{t}+\frac{2(-\rho)}{\left(|\partial \rho|_{\rho}^{2}-\rho\right)^{2}} r_{s}(u) R u^{s} r_{t}(u) \bar{R} u^{t}
$$

Since $r[u]=0$ on $\partial \Omega_{m}$, and $X_{i}$ is a tangential vector field, we have that

$$
0=X_{i} r[u]\left(z_{0}\right)=r_{s}(u) X_{i} u^{s}+r_{\bar{s}}(u) X_{i} \bar{u}^{s}=r_{s}(u) X_{i} u^{s}+\overline{r_{s}(u) \bar{X}_{i} u^{s}} .
$$

Thus,

$$
r_{s}(u) X_{i} u^{s} r_{t}(u) \bar{X}_{i} u^{t}\left(z_{0}\right)=-\left|\sum_{s=1}^{n} r_{s}(u) X_{i} u^{s}\left(z_{0}\right)\right|^{2}=-\left|\sum_{s=1}^{n} r_{s}(u) \bar{X}_{i} u^{s}\left(z_{0}\right)\right|^{2}
$$

for all $1 \leq i \leq m$. For any $z_{0} \in \partial \Omega_{m}$, by a rotation, we may assume $H(\rho)\left(z_{0}\right)$ is diagonal. Since $H(\rho)$ is positive definite, there is a positive constant $\epsilon$ so that $H(\rho) \geq \epsilon I_{n}$ for all $z \in \bar{\Omega}_{m}$. Therefore,

$$
\begin{aligned}
r_{s}(u) E^{s}[u]\left(z_{0}\right) & =-2 \sum_{i=1}^{m} \rho_{i \bar{i}}\left(z_{0}\right)\left|\sum_{s=1}^{n} r_{s}(u) X_{i} u^{s}\left(z_{0}\right)\right|^{2} \\
& =-2 \sum_{i=1}^{m} \rho_{i \bar{i}}\left(z_{0}\right)\left|\sum_{s=1}^{n} r_{s}(u) \bar{X}_{i} u^{s}\left(z_{0}\right)\right|^{2} .
\end{aligned}
$$

Since $u$ is proper harmonic $\left(\tau^{s}[u]=0\right.$ in $\left.\Omega_{m}\right)$, and $u \in C^{2}\left(\bar{\Omega}_{m}\right)$, one can easily see that $\sum_{s=1}^{n} r_{s}(u) E^{s}[u]=0$ on $\partial \Omega_{m}$. Combining this with the above identity, we obtain

$$
\sum_{s} r_{s}(u) X_{i} u^{s}\left(z_{0}\right)=\sum_{s=1}^{n} r_{s}(u) \bar{X}_{i} u^{s}=0, \quad 1 \leq i \leq m
$$

for all $z_{0} \in \partial \Omega_{m}$.

By the fact that $X_{1}, \cdots, X_{m}$ generate $T^{1,0}\left(\partial \Omega_{m}\right)$, we conclude that

$$
\sum_{s=1}^{n} r_{s}(u) \bar{\partial}_{b} u^{s}(z)=\sum_{s=1}^{n} r_{s}(u) \partial_{b} u^{s}(z)=0 \quad \text { for all } z \in \partial \Omega_{m} .
$$


The proof of the lemma is complete. $\quad$ ]

Let

$$
Y_{i}=\left(\delta_{i j}-\frac{\rho_{i} \rho^{j}}{|\partial \rho|_{\rho}^{2}-\rho}\right) \partial_{j}
$$

Note that $Y_{i}=\sum_{j} \rho_{i \bar{j}} X_{j}$. Thus $Y_{i} \in T^{(1,0)}\left(\partial \Omega_{m}\right)$. The following lemma expresses $\mathcal{L}$ in terms of the vector fields $X_{j}, \bar{X}_{j}, Y_{j}, \bar{Y}_{j}, R$ and $\bar{R}$.

Lemma 3.3. With the notation above, we have

$$
\begin{aligned}
\mathcal{L} & =X_{j} \bar{Y}_{j}+\frac{\mathcal{L}(\rho)}{|\partial \rho|_{\rho}^{2}-\rho} \bar{R}-\frac{\rho}{|\partial \rho|_{\rho}^{2}-\rho} R \frac{1}{|\partial \rho|_{\rho}^{2}-\rho} \bar{R} \\
& =\bar{X}_{j} Y_{j}+\frac{\mathcal{L}(\rho)}{|\partial \rho|_{\rho}^{2}-\rho} R-\frac{\rho}{|\partial \rho|_{\rho}^{2}-\rho} \bar{R} \frac{1}{|\partial \rho|_{\rho}^{2}-\rho} R
\end{aligned}
$$

Proof. The proof is just a simple computation. By definition

$$
\begin{aligned}
\mathcal{L} & =\sum_{i j}\left(\rho^{i \bar{j}}-\frac{\rho^{i} \rho^{\bar{j}}}{|\partial \rho|_{\rho}^{2}-\rho}\right) \partial_{i \bar{j}} \\
& =\sum_{j} X_{j} \partial_{\bar{j}} \\
& =\sum_{j} X_{j} \bar{Y}_{j}+\sum_{j} X_{j}\left(\frac{\rho^{\bar{\ell}} \rho_{\bar{j}}}{|\partial \rho|_{\rho}^{2}-\rho} \partial_{\bar{\ell}}\right) \\
& =\sum_{j} X_{j} \bar{Y}_{j}+\sum_{j, \ell=1}^{m} \frac{X_{j}\left(\rho_{\bar{j}}\right)}{|\partial \rho|_{\rho}^{2}-\rho} \rho^{\bar{\ell}} \partial_{\bar{\ell}}+\sum_{j, \ell=1}^{m} \rho_{\bar{j}} X_{j}\left(\frac{\rho^{\bar{\ell}}}{|\partial \rho|_{\rho}^{2}-\rho} \partial_{\bar{\ell}}\right) \\
& =\sum_{j} X_{j} \bar{Y}_{j}+\frac{\mathcal{L}(\rho)}{|\partial \rho|_{\rho}^{2}-\rho} \bar{R}+\frac{-\rho}{|\partial \rho|_{\rho}^{2}-\rho} R \frac{1}{|\partial \rho|_{\rho}^{2}-\rho} \bar{R}
\end{aligned}
$$

Similarly, we have

$$
\begin{aligned}
\mathcal{L} & =\sum_{i j}\left(\rho^{i \bar{j}}-\frac{\rho^{i} \rho^{\bar{j}}}{|\partial \rho|_{\rho}^{2}-\rho}\right) \partial_{i \bar{j}} \\
& =\sum_{j} \bar{X}_{j} \partial_{j} \\
& =\sum_{j} \bar{X}_{j} Y_{j}+\sum_{j} \bar{X}_{j}\left(\frac{\rho^{\ell} \rho_{j}}{|\partial \rho|_{\rho}^{2}-\rho} \partial_{\ell}\right) \\
& =\sum_{j} \bar{X}_{j} Y_{j}+\sum_{j, \ell=1}^{m} \frac{\bar{X}_{j}\left(\rho_{j}\right)}{|\partial \rho|_{\rho}^{2}-\rho} \rho^{\ell} \partial_{\ell}+\sum_{j, \ell=1}^{m} \rho_{j} \bar{X}_{j}\left(\frac{\rho^{\ell}}{|\partial \rho|_{\rho}^{2}-\rho} \partial_{\ell}\right) \\
& =\sum_{j} \bar{X}_{j} Y_{j}+\frac{\mathcal{L}(\rho)}{|\partial \rho|_{\rho}^{2}-\rho} R-\frac{\rho}{|\partial \rho|_{\rho}^{2}-\rho} \bar{R} \frac{1}{|\partial \rho|_{\rho}^{2}-\rho} R .
\end{aligned}
$$

The proof is complete. $\quad$ Q 
Since $Y_{i} \in T^{(1,0)}\left(\partial \Omega_{m}\right)$, it follows from Lemma 3.2 that

$$
\sum_{s=1}^{n} r_{s}(u) Y_{j} u^{s}(z)=0 \quad \text { on } \partial \Omega_{m}, \quad 1 \leq j \leq m .
$$

A similar reasoning shows that

$$
\sum_{s=1}^{n} r_{s}(u) \bar{Y}_{j} u^{s}(z)=0 \text { on } \partial \Omega_{m}, \quad 1 \leq j \leq m .
$$

This implies that for each $1 \leq j \leq m$ we have that

$$
0=\bar{X}_{j}\left(r_{s}(u) Y_{j} u^{s}\right)=r_{s t} \bar{X}_{j} u^{t} Y_{j} u^{s}+r_{s \bar{t}} \bar{X}_{j} \bar{u}^{t} Y_{j} u^{s}+r_{s}(u) \bar{X}_{j} Y_{j} u^{s}
$$

and

$$
0=X_{j}\left(r_{s}(u) \bar{Y}_{j} u^{s}\right)=r_{s t} X_{j} u^{t} \bar{Y}_{j} u^{s}+r_{s \bar{t}} X_{j} \bar{u}^{t} \bar{Y}_{j} u^{s}+r_{s}(u) X_{j} \bar{Y}_{j} u^{s} .
$$

Thus

$$
-r_{s}(u) \bar{X}_{j} Y_{j} u^{s}=r_{s \bar{t}}(u) \bar{X}_{j} \bar{u}^{t} Y_{j} u^{s}+r_{s t}(u) \bar{X}_{j} u^{t} Y_{j} u^{s}
$$

and

$$
-r_{s}(u) X_{j} \bar{Y}_{j} u^{s}=r_{s \bar{t}}(u) X_{j} \bar{u}^{t} \bar{Y}_{j} u^{s}+r_{s t}(u) X_{j} u^{t} \bar{Y}_{j} u^{s} .
$$

Therefore, it follows from Lemma 3.3 that on $\partial \Omega_{m}$

$$
\begin{aligned}
& \frac{\mathcal{L}(\rho)}{|\partial \rho|_{\rho}^{2}} \sum_{s} r_{s}(u) R u^{s} \\
& =\sum_{s=1}^{n} r_{s}(u) \mathcal{L} u^{s}-\sum_{s=1}^{n} \sum_{j=1}^{m} r_{s}(u) \bar{X}_{j} Y_{j} u^{s}+r_{s}(u) \frac{\rho(z)}{|\partial \rho|_{\rho}^{2}-\rho} \bar{R} \frac{1}{|\partial \rho|_{\rho}^{2}-\rho} R u^{s} \\
& =r_{s}(u) \mathcal{L} u^{s}(z)+r_{s \bar{t}}(u) \bar{X}_{j} \bar{u}^{t} Y_{j} u^{s}+r_{s t}(u) \bar{X}_{j} u^{t} Y_{j} u^{s} \\
& =r_{s}(u) \mathcal{L} u^{s}(z)+r_{s t}(u) \bar{X}_{j} u^{t} Y_{j} u^{s}+r_{s \bar{t}}(u) \bar{X}_{j} \bar{u}^{t} Y_{j} u^{s} .
\end{aligned}
$$

Similarly,

$$
\begin{aligned}
& \frac{\mathcal{L}(\rho)}{|\partial \rho|_{\rho}^{2}} \sum_{s} r_{s}(u) \bar{R} u^{s} \\
& =\sum_{s=1}^{n} r_{s}(u) \mathcal{L} u^{s}-\sum_{s=1}^{n} \sum_{j=1}^{m} r_{s}(u) X_{j} \bar{Y}_{j} u^{s}+r_{s}(u) \frac{\rho(z)}{|\partial \rho|_{\rho}^{2}-\rho} R \frac{1}{|\partial \rho|_{\rho}^{2}-\rho} \bar{R} u^{s} \\
& =r_{s}(u) \mathcal{L} u^{s}(z)+r_{s t}(u) X_{j} u^{t} \bar{Y}_{j} u^{s}+r_{s \bar{t}}(u) X_{j} \bar{u}^{t} \bar{Y}_{j} u^{s} .
\end{aligned}
$$

Recall that $a[u](z)$ is given by

$$
r(u)=a[u](z) \rho(z), \quad z \in \bar{\Omega}_{m} .
$$

It is easy to see that $a[u] \geq 0$ on $\bar{\Omega}_{m}, a[u](z)>0$ on $\Omega_{m}$ and $a[u] \in C^{1}\left(\bar{\Omega}_{m}\right)$. Thus, 


$$
\begin{aligned}
\tau^{s}[u]= & (-\rho) \mathcal{L} u^{s}+(-\rho)\left[r^{s \bar{\ell}}(u) r_{t \gamma \bar{\ell}}+\frac{r_{t \gamma}(u) r^{s}(u)-r^{s} r^{\bar{\ell}} r_{t \gamma \bar{\ell}}}{|\partial r|_{r}^{2}-r}\right] X_{j} u^{t} u \frac{\gamma}{\bar{j}} \\
& +\frac{\rho(z)}{r(u)} E^{s}[u] \\
= & (-\rho) \mathcal{L} u^{s}+(-\rho)\left[r^{s \bar{\ell}}(u) r_{t \gamma \bar{\ell}}(u)+\frac{r_{t \gamma}(u) r^{s}(u)-r^{s} r^{\bar{\ell}} r_{t \gamma \bar{\ell}}}{|\partial r|_{r}^{2}-r}\right] X_{j} u^{t} u_{\bar{j}}^{\gamma} \\
& +\frac{1}{a[u]} \rho_{i \bar{j}}\left[X_{i} u^{s} r_{t}(u) \bar{X}_{j} u^{t}+\bar{X}_{j} u^{s} r_{t}(u) X_{i} u^{t}\right] \\
& +\frac{1}{a[u]} \frac{(-\rho)}{\left(\left|\partial \rho_{\rho}\right|^{2}-\rho\right)^{2}}\left[R u^{s} r_{t}(u) \bar{R} u^{t}+\bar{R} u^{s} r_{t}(u) R u^{t}\right] \\
= & (-\rho)\left[\mathcal{L} u^{s}+\left(r^{s \bar{\ell}}(u) r_{t \gamma \bar{\ell}}+\frac{r_{t \gamma}(u) r^{s}(u)-r^{s}(u) r^{\bar{\ell}}(u) r_{t \gamma \bar{\ell}}(u)}{|\partial r|_{r}^{2}-r}\right) X_{j} u^{t} u_{\bar{j}}^{\gamma}\right. \\
& \left.+\frac{1}{a[u]} \frac{1}{\left(|\partial \rho|_{\rho}^{2}-\rho\right)^{2}}\left(R u^{s} r_{t}(u) \bar{R} u^{t}+\bar{R} u^{s} r_{t}(u) R u^{t}\right)\right] \\
& +\frac{1}{a[u]} \rho_{i \bar{j}}\left[X_{i} u^{s} r_{t}(u) \bar{X}_{j} u^{t}+\bar{X}_{j} u^{s} r_{t}(u) X_{i} u^{t}\right] .
\end{aligned}
$$

Since $\sum_{s=1}^{n} r_{s}(u) r^{s}(u)=|\partial r|_{r}^{2}$, we have that on $\partial \Omega_{m}$

$$
\begin{aligned}
& \sum_{s=1}^{n}\left(r^{s \bar{\ell}}(u) r_{t \gamma \bar{\ell}}+\frac{r_{t \gamma}(u) r^{s}(u)-r^{s}(u) r^{\bar{\ell}} r_{t \gamma \bar{\ell}}}{|\partial r|_{r}^{2}}\right) r_{s}(u) X_{j} u^{t} u \frac{\gamma}{j} \\
& =\left(r^{\bar{\ell}}(u) r_{t \gamma \bar{\ell}}+r_{t \gamma}(u)-r^{\bar{\ell}} r_{t \gamma \bar{\ell}}(u)\right) X_{j} u^{t} u \frac{\gamma}{\bar{j}} \\
& =r_{t \gamma}(u) X_{j} u^{t} u \frac{\gamma}{\bar{j}} .
\end{aligned}
$$

Thus on $\partial \Omega_{m}$,

$$
\begin{aligned}
r_{s}(u) \tau^{s}[u] & =\frac{2}{a[u]} \rho_{i \bar{j}}\left(r_{s}(u) X_{i} u^{s}\right)\left(r_{t}(u) \bar{X}_{j} u^{t}\right) \\
& +(-\rho)\left[\left(r_{s}(u) \mathcal{L} u^{s}+r_{t \gamma}(u) X_{j} u^{t} u \frac{\gamma}{j}\right)+2 \frac{r_{s}(u) R u^{s} r_{t}(u) \bar{R} u^{t}}{a[u]\left(|\partial \rho|_{\rho}^{2}-\rho\right)^{2}}\right] .
\end{aligned}
$$

Since $u \in C^{2}\left(\bar{\Omega}_{m}\right)$, we know by Lemma 3.2 that

$$
\left(\sum_{s} r_{s}(u) X_{i} u^{s}\right)\left(\sum_{s} r_{s}(u) \bar{X}_{j} u^{s}\right)=O\left(\rho(z)^{2}\right) .
$$

From the harmonicity of $u$, it follows that $r_{s}(u) \tau^{s}[u]=0$. This implies that for any $z \in \partial \Omega_{m}$

$$
a[u]\left(r_{s}(u) \mathcal{L} u^{s}+r_{t \gamma}(u) X_{j} u^{t} u_{j}^{\gamma}\right)+2 \frac{r_{s}(u) R u^{s} r_{t}(u) \bar{R} u^{t}}{|\partial \rho|_{\rho}^{4}}=0
$$

Theorem 3.4. Let $\Omega_{m} \subset \mathbb{C}^{m}(m>1)$ and $\Omega_{n} \subset \mathbb{C}^{n}$ be smoothly bounded strictly pseudoconvex domains with metric $h$ and $g$, respectively. Let $u=\left(u^{1}, \cdots, u^{n}\right) \in$ $C^{2}\left(\bar{\Omega}_{m}\right)$ be a proper harmonic map from $\left(\Omega_{m}, h\right)$ to $\left(\Omega_{n}, g\right)$. If

$$
\lim _{w \rightarrow z}\left(r_{s} \mathcal{L} u^{s}(w)+r_{s t}(u) X_{j} u^{t} u \frac{s}{j}\right)=0, \quad \text { for } z \in \partial \Omega_{m},
$$


then either $u$ is $C R$ or $\bar{u}$ is $C R$.

Proof. Notice that

$$
\sum_{j=1}^{m} \rho_{j} X_{j}=\frac{(-\rho) R}{|\partial \rho|_{\rho}^{2}-\rho}
$$

By a rotation if necessary, we may assume without loss of generality that for any $z_{0} \in \partial \Omega_{m}$, we have that $\rho_{i \bar{j}}\left(z_{0}\right)=\rho_{i \bar{i}}\left(z_{0}\right) \delta_{i j}$. Therefore,

$$
\begin{aligned}
r_{s t}(u) X_{j} u^{t} u \frac{s}{j}\left(z_{0}\right) & =r_{s t}(u) X_{j} u^{t} \bar{Y}_{j} u^{s}\left(z_{0}\right)+\frac{r_{s t}(u) X_{j} u^{t} \rho_{j} \rho^{k} \partial_{\bar{k}} u^{s}\left(z_{0}\right)}{|\partial \rho|_{\rho}^{2}-\rho} \\
& =r_{s t}(u) X_{j} u^{t} \bar{Y}_{j} u^{s}\left(z_{0}\right) .
\end{aligned}
$$

Now (3.26) and (3.27) imply that

$$
\left(r_{s}(u) R u^{s}\left(z_{0}\right)\right)\left(r_{t}(u) \bar{R} u^{t}\left(z_{0}\right)\right)=0
$$

Also

$$
\begin{aligned}
Y_{j} u^{s}\left(z_{0}\right) & =\rho_{j \bar{j}}\left(z_{0}\right)\left(\rho^{j \bar{j}} \partial_{j}-\rho^{j \bar{j}} \frac{\rho_{j} \rho^{k}}{|\partial \rho|_{\rho}^{2}} \partial_{k}\right) u^{s}\left(z_{0}\right) \\
& =\rho_{j \bar{j}}\left(z_{0}\right)\left(\rho^{k \bar{j}} \partial_{k}-\frac{\rho^{\bar{j}} \rho^{k}}{|\partial \rho|_{\rho}^{2}} \partial_{k}\right) u^{s}\left(z_{0}\right) \\
& =\rho_{j \bar{j}}\left(z_{0}\right) X_{j} u^{s}\left(z_{0}\right) .
\end{aligned}
$$

Similarly, we have

$$
\bar{Y}_{j} u^{s}\left(z_{0}\right)=\rho_{j \bar{j}}\left(z_{0}\right) \bar{X}_{j} u^{s}\left(z_{0}\right) .
$$

Combining (3.29) with (3.22) and (3.23), and using (3.30) and (3.31), we obtain

$$
\left(r_{s \bar{t}} \rho_{j \bar{j}} \bar{X}_{j} u^{s} X_{j} \bar{u}^{t}\right)\left(r_{p \bar{q}} \rho_{k \bar{k}} X_{k} u^{s} \bar{X}_{k} \bar{u}^{t}\right)=0 .
$$

Since $\left(r_{p \bar{q}}\left(u\left(z_{0}\right)\right)\right.$ and $H(\rho)\left(z_{0}\right)$ are positive definite, we have

$$
\left[\sum_{j=1}^{m} \sum_{s=1}^{n}\left|X_{j} u^{s}(z)\right|^{2}\right]\left[\sum_{j=1}^{m} \sum_{t=1}^{n}\left|\bar{X}_{j} u^{t}\left(z_{0}\right)\right|^{2}\right]=0 .
$$

Therefore, either $\bar{\partial}_{b} \bar{u}^{s}\left(z_{0}\right)=0$ or $\partial_{b} \bar{u}^{s}\left(z_{0}\right)=0$ for all $1 \leq s \leq n$. The proof is complete by applying Theorem 2.1. $\quad$ प

The following lemma gives an expression for $a[u](z)$ in terms of the vector fields $R$ and $\bar{R}$ and provides a sufficient condition for two proper harmonic maps with the same boundary data to be equal.

LEMMA 3.5. Let $u: \Omega_{m} \rightarrow \Omega_{n}$ be a proper harmonic map so that $u \in C^{2}\left(\bar{\Omega}_{m}\right)$.

(i) Then $r_{s}(u) R u^{s}$ and $r_{s}(u) \bar{R} u^{s}$ are non-negative on $\partial \Omega_{m}$. In particular, for any $z \in \partial \Omega_{m}$ with $|\partial \rho|_{0}^{2}=\sum_{j=1}^{n}\left|\partial_{j} \rho\right|^{2}$, we have

$$
a[u](z)|\partial \rho|_{0}^{2}=\frac{r_{s}(u)(R+\bar{R}) u^{s}(z)}{|\partial \rho|_{\rho}^{2}} .
$$


(ii) On $\left\{z \in \partial \Omega_{m}: a[u](z)>0\right\}$ we have

$$
\begin{gathered}
r_{s} \mathcal{L} u^{s}(w)+r_{s t}(u) X_{j} u^{t} u_{j}^{s}=\frac{-(m+1) E[u](z)+D[u](z)}{4 m} \\
a[u](z)|\partial \rho|_{0}^{2}=\frac{E(u)(z)}{2 m}+\frac{D[u]}{2 m(m-1)}
\end{gathered}
$$

where

$$
E[u](z)=r_{s \bar{t}}(u) \overline{X_{j} u^{t}} Y_{j} u^{s}\left(z_{0}\right)+r_{s \bar{t}}(u) \overline{\bar{X}_{j} u^{t}} \bar{Y}_{j} u^{s}\left(z_{0}\right)
$$

and

$$
D[u]=\left[(m+1)^{2} E[u]^{2}-16 m\left(r_{s \bar{t}}(u) \overline{X_{j} u^{t}} Y_{j} u^{s}\left(z_{0}\right)\right)\left(r_{s \bar{t}}(u) \overline{\bar{X}}_{j} u^{t} \bar{Y}_{j} u^{s}\left(z_{0}\right)\right)\right]^{1 / 2} .
$$

(iii) For $z \in \partial \Omega_{m}$, we have that $E_{b}(u)(z)>0$ if and only if $a[u](z)>0$.

(iv) If $u(z)=v(z)$ and $a[u](z)>0$ on $\partial \Omega_{m}$, then $u \equiv v$ on $\Omega_{m}$.

Proof. For any $z_{0} \in \partial \Omega_{m}$, we may assume that $\left|\partial \rho\left(z_{0}\right)\right|^{2}=1$; otherwise, we may use $\tilde{\rho}(z)=\rho(z) /\left|\partial \rho\left(z_{0}\right)\right|$ to replace $\rho$ and use $\tilde{r}(w)=r(w) /\left|\partial \rho\left(z_{0}\right)\right|_{0}$ to replace $r(w)$. By a rotation if necessary, we may assume without loss of generality that $H(\rho)\left(z_{0}\right)$ is diagonal.

First we prove (i). Let

$$
\begin{gathered}
A=\sum_{s=1}^{n} r_{s}(u) \mathcal{L} u^{s}\left(z_{0}\right)+\sum_{s, t=1}^{n} r_{s t}(u) u \frac{t}{j} X_{j} u^{s}\left(z_{0}\right), \\
A_{1}=r_{s}(u) R u^{s}\left(z_{0}\right), \quad A_{2}=r_{s}(u) \bar{R} u^{s}\left(z_{0}\right) \\
E_{1}=r_{s \bar{t}}(u) \overline{X_{j} u^{t}} Y_{j} u^{s}\left(z_{0}\right) \\
=r_{s \bar{t}}(u) \rho_{j \bar{j}} X_{j} u^{s} \bar{X}_{j} u^{t}\left(z_{0}\right), \text { and } \\
E_{2}=r_{s \bar{t}}(u) \overline{\bar{X}}_{j} u^{t} \bar{Y}_{j} u^{s}\left(z_{0}\right) \\
=r_{s \bar{t}}(u) \rho_{j \bar{j}} \bar{X}_{j} u^{s} \overline{\bar{X}}_{j} u^{t}\left(z_{0}\right)
\end{gathered}
$$

since $Y_{j} u^{s}=\rho_{j \bar{j}} X_{j} u^{s}$ by $(3.30)$.

Then by (3.22)

$$
\frac{\mathcal{L}(\rho)}{|\partial \rho|_{\rho}^{2}} A_{1}=A+r_{s \bar{t}}(u) \rho_{j \bar{j}} X_{j} u^{s} \overline{X_{j} u^{t}}=A+E_{1},
$$

by $(3.23)$

$$
\frac{\mathcal{L}(\rho)}{|\partial \rho|_{\rho}^{2}} A_{2}=A+r_{s \bar{t}}(u) \rho_{j \bar{j}} \bar{X}_{j} u^{s} \overline{\bar{X}_{j} u^{t}}=A+E_{2},
$$

and by (3.26)

$$
a[u] A+\frac{2}{|\partial \rho|_{\rho}^{4}} A_{1} A_{2}=0
$$


Thus,

$$
\mathcal{L}(\rho)^{2} a[u] A+2\left[A+E_{1}\right]\left[A+E_{2}\right]=0
$$

or

$$
2 A^{2}+\left(2 E_{1}+2 E_{2}+\mathcal{L}(\rho)^{2} a[u]\right) A+2 E_{1} E_{2}=0 .
$$

Therefore, $A$ is non-positive since $E_{j}$ is non-negative for $j=1,2$. Thus $A_{j}$ is real for $j=1,2$. Moreover, $A<0$ when both $E_{1}>0$ and $E_{2}>0$.

Define $\mathcal{R}=\rho_{\bar{j}} \partial_{j}, \overline{\mathcal{R}}=\rho_{j} \partial_{\bar{j}}$. Since $(\mathcal{R}-\overline{\mathcal{R}}) r(u(z))=0$ for $z \in \partial \Omega_{m}$, we have that

$$
a[u]=\frac{\mathcal{R}+\overline{\mathcal{R}}}{2} r(u)=\mathcal{R} r(u)=\overline{\mathcal{R}} r(u) .
$$

Also $R=\langle R, \mathcal{R}\rangle \mathcal{R}+T$ where $T$ is a tangential vector. Since $\langle R, \mathcal{R}\rangle=|\partial \rho|_{\rho}^{2}$

$$
a[u]=\mathcal{R} r(u)=\frac{\operatorname{Rr}(u)}{|\partial \rho|_{\rho}^{2}}-\frac{\operatorname{Tr}(u)}{|\partial \rho|_{\rho}^{2}}=\frac{\operatorname{Rr}(u)}{|\partial \rho|_{\rho}^{2}}
$$

and

$$
a[u]=\overline{\mathcal{R}} r(u)=\frac{\bar{R} r(u)}{|\partial \rho|_{\rho}^{2}}-\frac{\bar{T} r(u)}{|\partial \rho|_{\rho}^{2}}=\frac{\bar{R} r(u)}{|\partial \rho|_{\rho}^{2}} .
$$

Since $A_{2}$ is real, we obtain

$$
\begin{aligned}
0 \leq a[u]=\frac{R r(u)}{|\partial \rho|_{\rho}^{2}} & =\frac{1}{|\partial \rho|_{\rho}^{2}}\left(r_{s}(u) R u^{s}+r_{\bar{s}}(u) R \bar{u}^{s}\right) \\
& =\frac{1}{|\partial \rho|_{\rho}^{2}}\left(r_{s}(u) R u^{s}+\overline{r_{s}(u) \bar{R} u^{s}}\right) \\
& =\frac{1}{|\partial \rho|_{\rho}^{2}}\left(A_{1}+\bar{A}_{2}\right) \\
& =\frac{1}{|\partial \rho|_{\rho}^{2}}\left(A_{1}+A_{2}\right),
\end{aligned}
$$

which is (3.32). Also by (3.39) and the nonpositivity of $A$,

$$
A_{1} A_{2}=-\frac{a[u] A|\partial \rho|_{\rho}^{4}}{2} \geq 0 .
$$

Thus we have established that $A_{1}+A_{2} \geq 0$ and $A_{1} A_{2} \geq 0$. As a result, $A_{j} \geq 0$ for $j=1,2$. This finishes the proof of (i).

Next we prove (ii). Using the fact that $\rho^{i} \rho^{\bar{j}} \rho_{i \bar{j}}=\rho^{i \bar{k}} \rho_{\bar{k}} \rho^{\bar{j}} \rho_{i \bar{j}}=\rho^{i \bar{k}} \rho_{i \bar{j}} \rho_{\bar{k}} \rho^{\bar{j}}=$ $\delta_{k j} \rho_{\bar{k}} \rho^{\bar{j}}=\rho_{\bar{j}} \rho^{\bar{j}}=|\partial \rho|_{\rho}^{2}$ we have that on $\partial \Omega_{m}$

$$
\begin{aligned}
\mathcal{L}(\rho) & =\sum_{i j}\left(\rho^{i \bar{j}}-\frac{\rho^{i} \rho^{\bar{j}}}{|d \rho|^{2}-\rho}\right) \rho_{i \bar{j}} \\
& =\sum_{i} \delta_{i i}-\sum_{i j} \frac{\rho^{i} \rho^{\bar{j}}}{|d \rho|^{2}-\rho} \rho_{i \bar{j}}
\end{aligned}
$$




$$
\begin{aligned}
& =m-\frac{|\partial \rho|_{\rho}^{2}}{|\partial \rho|_{\rho}^{2}-\rho} \\
& =m-1 .
\end{aligned}
$$

Adding (3.37) to (3.38) and using (3.41) together with (3.42), we have that

$$
a[u]=\frac{2 A+E[u]}{m-1} \text { since } E[u]=E_{1}+E_{2} .
$$

Substituting (3.43) into (3.40) and using (3.42), we obtain

$$
m A^{2}+\left(\frac{m+1}{2}\right) E[u] A+E_{1} E_{2}=0
$$

Since $A_{i} \geq 0$ for $i=1,2,(3.37)$ and (3.38) implies that if $a[u]>0$ or $E_{1} \neq E_{2}$, then

$$
A=\frac{-(m+1) E[u]+D[u]}{4 m}
$$

where $D[u]=\sqrt{(m+1)^{2} E[u]^{2}-16 m E_{1} E_{2}}$. This establishes (3.33). By (3.43) and $(3.45)$

$$
\begin{aligned}
a[u] & =\frac{2 A+E[u]}{m-1} \\
& =\frac{1}{m-1}\left(\frac{-(m+1) E[u]+D[u]}{2 m}+E[u]\right) \\
& =\frac{E[u]}{2 m}+\frac{D[u]}{2 m(m-1)}
\end{aligned}
$$

which is (3.34).

Now we prove (iii). By (ii) if $a[u]>0$, then $E[u]>0$ which implies that $E_{b}(u)>0$ since $r_{s \bar{t}}$ is positive definite. In order to establish the converse, we must show that for every $z_{0} \in \partial \Omega_{m}$ such that $a[u]\left(z_{0}\right)=0$, it holds that $E_{b}(u)\left(z_{0}\right)=0$. Since $r_{s \bar{t}}$ is positive definite, this is equivalent to showing that $E[u]=0$. If $z_{0}$ is a boundary point of the zero set of $a[u]$ on $\partial \Omega_{m}$, then $E[u]=0$ by (3.34) and passing to the limit. So assume that $z_{0}$ is an interior point of the zero set of $a[u]$ on $\partial \Omega_{m}$. Since $r(u)=a[u] \rho(z)$, we have that $\operatorname{Rr}(u)=\operatorname{Ra}[u] \rho(z)+a[u] R \rho(z)$. Therefore,

$$
R r(u)=r_{s} R u^{s}+r_{\bar{s}} R \bar{u}^{s}=r_{s} R u^{s}+\overline{r_{s} \bar{R} u^{s}}=R a[u] \rho(z)+a[u]|\partial \rho|_{\rho}^{2} .
$$

Thus,

$$
\begin{aligned}
& 2 \operatorname{Re}\left(r_{s} R u^{s}\right)\left(r_{s} \bar{R} u^{s}\right) a[u]^{-1} \\
& =\left.\left.|\operatorname{Ra}[u] \rho(z)+a[u]| \partial \rho\right|_{\rho} ^{2}\right|^{2} a[u]^{-1}-\left(\left|r_{s} R u^{s}\right|^{2}+\left|r_{\bar{s}} R \bar{u}^{s}\right|^{2}\right) a[u]^{-1} \\
& \leq\left.\left.|\operatorname{Ra}[u] \rho(z)+a[u]| \partial \rho\right|_{\rho} ^{2}\right|^{2} a[u]^{-1} \\
& =|\operatorname{Ra}[u]|^{2} \rho(z)^{2} a[u]^{-1}+a[u]|\partial \rho|_{\rho}^{4}+2 \operatorname{Re}\left(\operatorname{Ra}[u] \rho(z)|\partial \rho|_{\rho}^{2}\right) \\
& =|\operatorname{Ra}[u]|^{2} \rho(z)^{2} a[u]^{-1}+a[u]|\partial \rho|_{\rho}^{4}+(R+\bar{R}) a[u] \rho(z)|\partial \rho|_{\rho}^{2} \\
& =4\left(\operatorname{Ra}[u]^{1 / 2}\right)\left(\bar{R} a[u]^{1 / 2}\right) \rho(z)^{2}+a[u]|\partial \rho|_{\rho}^{4}+(R+\bar{R}) a[u] \rho(z)|\partial \rho|_{\rho}^{2} .
\end{aligned}
$$

Since $X_{i} r(u)=X_{i} a[u] \rho(z)+a[u] X_{i}(\rho)$ and $X_{i}(\rho)=\frac{-\rho \rho^{\bar{i}}}{|\partial \rho|_{\rho}^{2}-\rho}$, we have

$$
X_{i} r(u)=r_{s} X_{i} u^{s}+\overline{r_{s} \bar{X}_{i} u^{s}}=X_{i} a[u] \rho(z)-a[u] \frac{\rho \rho^{\bar{i}}}{|\partial \rho|_{\rho}^{2}-\rho} .
$$


Thus,

$$
\begin{aligned}
& 2 \operatorname{Re}\left[\left(r_{s} X_{i} u^{s}\right)\left(r_{s} \bar{X}_{i} u^{s}\right) a[u]^{-1} \rho(z)^{-1}\right. \\
& =\left|X_{i} a[u] \rho(z)-a[u] \frac{\rho \rho^{\bar{i}}}{|\partial \rho|_{\rho}^{2}-\rho}\right|^{2} a[u]^{-1} \rho(z)^{-1}-\frac{\left(\left|r_{s} X_{i} u^{s}\right|^{2}+\left|r_{s} \bar{X}_{i} u^{s}\right|^{2}\right)}{a[u] \rho(z)} \\
& \leq\left|X_{i} a[u] \rho(z)-a[u] \frac{\rho \rho^{\bar{i}}}{|\partial \rho|_{\rho}^{2}-\rho}\right|^{2} a[u]^{-1} \rho(z)^{-1} \\
& =\left|X_{i} a[u]\right|^{2} \rho(z) a[u]^{-1}-\frac{2 \operatorname{Re}\left(X_{i} a[u] \rho^{i}\right)}{|\partial \rho|_{\rho}^{2}-\rho} \rho+\frac{\left|\rho^{\bar{i}}\right|^{2} a[u] \rho(z)}{\left(|\partial \rho|_{\rho}^{2}-\rho\right)^{2}} \\
& =4\left(X_{i} a[u]^{1 / 2}\right)\left(\bar{X}_{i} a[u]^{1 / 2}\right) \rho-\frac{2 \operatorname{Re}\left(X_{i} a[u] \rho^{i}\right)}{|\partial \rho|_{\rho}^{2}-\rho} \rho+\frac{\left|\rho^{\bar{i}}\right|^{2} a[u] \rho(z)}{\left(|\partial \rho|_{\rho}^{2}-\rho\right)^{2}} .
\end{aligned}
$$

Since $a[u] \in C^{1}\left(\bar{\Omega}_{m}\right)$ and $a[u]=0$ on $\partial \Omega_{m} \cap B\left(z_{0}, \delta\right)$ for some $\delta>0$, it is clear that $a[u]^{1 / 2} \in C^{1 / 2}\left(\bar{\Omega}_{m}\right)$. Therefore,

$$
\lim _{z \rightarrow z_{0}}\left[\left(R a[u]^{1 / 2}\right)\left(\bar{R} a[u]^{1 / 2}\right) \rho(z)^{2}\right]=0 .
$$

At the same time, since $X_{i}$ and $\bar{X}_{i}$ are tangential

$$
\lim _{z \rightarrow z_{0}}\left[\left(X_{i} a[u]^{1 / 2}\right)\left(\bar{X}_{i} a[u]^{1 / 2}\right) \rho(z)\right]=0 .
$$

Thus, (3.25) and the previous computations show that

$$
\begin{aligned}
0= & \limsup _{z \rightarrow z_{0}}\left[\operatorname{Re}\left(r_{s}(u) \mathcal{L} u^{s}+r_{s t}(u) X_{j} u^{t} u \frac{s}{j}\right)+2 \operatorname{Re} \frac{r_{s}(u) R u^{s} r_{t}(u) \bar{R} u^{t}}{a[u] \mid\left(\left.d \rho\right|^{2}-\rho\right)^{2}}\right. \\
& \left.+\frac{2 \operatorname{Re}}{a[u]|\rho|} \rho_{i \bar{j}}\left(r_{s}(u) X_{i} u^{s}\right)\left(r_{t}(u) \bar{X}_{j} u^{t}\right)\right] \\
= & \limsup _{z \rightarrow z_{0}} \operatorname{Re}\left(r_{s}(u) \mathcal{L} u^{s}+r_{s t}(u) X_{j} u^{t} u \frac{s}{j}\right) .
\end{aligned}
$$

This implies that $\left(r_{s}(u) \mathcal{L} u^{s}+r_{s t}(u) X_{j} u^{t} u \frac{s}{j}\right) \geq 0$ at $z_{0}$. By (3.43), we know that $0=a[u]=\frac{2 A+E[u]}{m-1}$. Thus $0 \leq 2 A=-E$ which implies that $E=0$.

Finally, we prove (iv). Since $a[u](z)>0$ for all $z \in \partial \Omega_{m}$, it follows from (ii) that $a[v]=a[u]$ on $\partial \Omega_{m}$. Next we show $d_{\Omega_{n}}(u(z), v(z))=0$ for $z \in \partial \Omega_{m}$. For each $z_{0} \in \partial \Omega_{m}$, after a holomorphically change of coordinates, we may assume that $z_{0}=0$, $w_{0}=u\left(z_{0}\right)=0$ and

$$
r(w)=-\lambda_{0} \operatorname{Re} w^{n}+\sum_{j=1}^{n} \lambda_{j}\left|w^{j}\right|^{2}+o\left(|w|^{2}\right), \quad \lambda_{j}>0
$$

for all $w \in \Omega_{n} \cap B(0, \epsilon)$ for some $0<\epsilon<<1$. For any $z \in \Omega_{m}$ with $|z| \leq \delta$ so that $u(z), v(z) \in \Omega_{n} \cap B(0, \epsilon)$, let $S=\left\{\gamma:[0,1] \rightarrow \Omega_{n}\right.$ : $\gamma$ is piecewise differentiable curve with $\gamma(0)=v(z), \gamma(1)=u(z)\}$. Then

$$
\begin{aligned}
d_{\Omega_{n}}(u(z), v(z)) & =\inf _{\gamma \in S} \int_{0}^{1} \sqrt{g_{i \bar{j}}(\gamma(t)) \gamma^{i^{\prime}}(t) \overline{\gamma^{j^{\prime}}(t)}} d t \\
& =\inf _{\gamma \in S} \int_{0}^{1} \sqrt{\left[\frac{1}{-r}\left(r_{i \bar{j}}+\frac{r_{i} r_{\bar{j}}}{-r}\right)\right](\gamma(t)) \gamma^{i^{\prime}(t) \overline{\gamma^{j^{\prime}}(t)}} d t}
\end{aligned}
$$




$$
\leq C \inf _{\gamma \in S} \int_{0}^{1}\left(\frac{\left|\gamma^{\prime}(t)\right|}{\sqrt{-r(\gamma(t))}}+\frac{\mid\left(r(\gamma(t))^{\prime} \mid\right.}{|r(\gamma(t))|}\right) d t
$$

Since $r(w)$ is convex in $\Omega_{n} \cap B(0, \epsilon)$ and assuming that $r(v(z))<r(u(z)$, we have that $r(t u(z)+(1-t) v(z))$ is an increasing convex function in $t$ and

$$
\begin{aligned}
a[t u(z)+(1-t) v(z)] & =\frac{r(t u(z)+(1-t) v(z))}{\rho(z)} \\
& \geq t a[u](z)+(1-t) a[v(z)] \\
& \geq a[u]\left(z_{0}\right) / 2
\end{aligned}
$$

when $\epsilon>0$ is small and $z \in \Omega_{n} \cap B(0, \epsilon)$. Let $\pi(z)$ be the radial projection of $z$ onto $\partial \Omega_{m}$. Then

$$
\begin{aligned}
|u(z)-v(z)| & \leq|u(z)-\phi(\pi(z))|+|\phi(\pi(z))-v(z)| \\
& =|u(z)-u(\pi(z))|+|v(z)-v(\pi(z))| \\
& \leq C\left(|u|_{1}+|v|_{1}\right)|\rho(z)| .
\end{aligned}
$$

Let

$$
\gamma(t)=t u(z)+(1-t) v(z) \in S
$$

Then

$$
\begin{aligned}
d_{\Omega_{n}}(u(z), v(z)) & \leq \frac{2 C}{\sqrt{a[u]\left(z_{0}\right)}} \int_{0}^{1} \frac{\left|\gamma^{\prime}(t)\right|}{\sqrt{-\rho(z))}} d t+C \int_{0}^{1} \frac{\left(r(\gamma(t))^{\prime}\right.}{-r(\gamma(t))} d t \\
& =\frac{2 C}{\sqrt{a[u]\left(z_{0}\right)}} \frac{|u(z)-v(z)|}{\sqrt{|\rho(z)|}}+C \log \frac{r(v(z))}{r(u(z))} \\
& \leq \frac{2 C}{\sqrt{a[u]\left(z_{0}\right)}} C\left(|u|_{1}+|v|_{1}\right) \sqrt{|\rho(z)|}+C \log \frac{a[v(z)]}{a[u(z)]} \\
& \rightarrow 0 \quad \text { as } z \rightarrow z_{0} .
\end{aligned}
$$

Using the fact established in [30] that $d_{\Omega_{n}}(u(z), v(z))$ is subharmonic whenever $u$ and $v$ are harmonic maps, we conclude by the maximum principle that $u=v$. Thus we have proved (iv). Therefore, the proof of the lemma is complete.

4. The energy density function. The goal of this section is to calculate the energy density function on $\partial \Omega_{m}$.

Lemma 4.1. Assume that $u \in C^{2}\left(\bar{\Omega}_{m}\right)$ is a harmonic map from $\Omega_{m}$ to $\Omega_{n}$. Then for any $z_{0} \in \Omega_{m}$ such that $a[u]\left(z_{0}\right)>0$, we have that

$$
\lim _{z \rightarrow z_{0}} e[u](z)=m+2 \frac{\left(r_{\alpha} R u^{\alpha}\right)\left(z_{0}\right)\left(r_{\beta} \bar{R} u^{\beta}\right)\left(z_{0}\right)}{\left(a[u]\left(z_{0}\right)\right)^{2}\left(|\partial \rho|_{\rho}^{4}\right)} .
$$

Proof. For any $z_{0} \in \partial \Omega_{m}$, we may assume that $\left|\partial \rho\left(z_{0}\right)\right|^{2}=1$; otherwise, we may use $\tilde{\rho}(z)=\rho(z) /\left|\partial \rho\left(z_{0}\right)\right|$ to replace $\rho$ and use $\tilde{r}(w)=r(w) /\left|\partial \rho\left(z_{0}\right)\right|_{0}$ to replace $r(w)$. By diagonalizing we can assume without loss of generality that at $z_{0} \in \partial \Omega_{m}$ we 
have that $\rho_{i \bar{j}}\left(z_{0}\right)=\delta_{i j} \rho_{j \bar{j}}\left(z_{0}\right), r_{\alpha \bar{\beta}}\left(u\left(z_{0}\right)\right)=\delta_{\alpha \beta} r_{\alpha \bar{\alpha}}\left(u\left(z_{0}\right)\right)$. By definition the energy density function equals

$$
\begin{aligned}
e & {[u]\left(z_{0}\right) } \\
= & h^{i \bar{j}} g_{\alpha \bar{\beta}}\left(\partial_{i} u^{\alpha} \overline{\partial_{j} u^{\beta}}+\partial_{\bar{j}} u^{\alpha} \overline{\partial_{\bar{i}} u^{\beta}}\right) \\
= & (-\rho)\left[\rho^{i \bar{j}}-\frac{\rho^{i} \rho^{\bar{j}}}{|\partial \rho|_{\rho}^{2}-\rho}\right]\left(\frac{1}{-r}\right)\left[r_{\alpha \bar{\beta}}+\frac{r_{\alpha} r_{\bar{\beta}}}{-r}\right]\left(\partial_{i} u^{\alpha} \overline{\partial_{j} u^{\beta}}+\partial_{\bar{j}} u^{\alpha} \overline{\partial_{\bar{i}} u^{\beta}}\right) \\
= & \left(\frac{\rho}{r}\right)\left\{X_{j} u^{\alpha} \overline{\partial_{j} u^{\beta}}+X_{j} \bar{u}^{\beta} \partial_{\bar{j}} u^{\alpha}\right\}\left[r_{\alpha \bar{\beta}}+\frac{r_{\alpha} r_{\bar{\beta}}}{-r}\right] \\
= & \left(\frac{\rho}{r}\right)\left\{X_{j} u^{\alpha} \overline{Y_{j} u^{\beta}}+X_{j} \bar{u}^{\beta} \bar{Y}_{j} u^{\alpha}+X_{j} u^{\alpha} \frac{\rho_{\bar{j}} \bar{R} \bar{u}^{\beta}}{|\partial \rho|_{\rho}^{2}-\rho}+X_{j} \bar{u}^{\beta} \frac{\rho_{\bar{j}} \bar{R} u^{\alpha}}{|\partial \rho|_{\rho}^{2}-\rho}\right\} \\
& \times\left[r_{\alpha \bar{\beta}}+\frac{r_{\alpha} r_{\bar{\beta}}}{-r}\right] \\
= & \left(\frac{\rho}{r}\right) r_{\alpha \bar{\beta}}\left\{X_{j} u^{\alpha} \overline{Y_{j} u^{\beta}}+X_{j} \bar{u}^{\beta} \bar{Y}_{j} u^{\alpha}+X_{j} u^{\alpha} \frac{\rho_{\bar{j}} \bar{R} \bar{u}^{\beta}}{|\partial \rho|_{\rho}^{2}-\rho}+X_{j} \bar{u}^{\beta} \frac{\rho_{\bar{j}} \bar{R} u^{\alpha}}{|\partial \rho|_{\rho}^{2}-\rho}\right\} \\
& +\left(\frac{\rho}{r}\right)\left(\frac{r_{\alpha} r_{\bar{\beta}}}{-r}\right)\left\{X_{j} u^{\alpha} \overline{Y_{j} u^{\beta}}+X_{j} \bar{u}^{\beta} \bar{Y}_{j} u^{\alpha}+X_{j} u^{\alpha} \frac{\rho_{\bar{j}} \bar{R} \bar{u}^{\beta}}{|\partial \rho|_{\rho}^{2}-\rho}+X_{j} \bar{u}^{\beta} \frac{\rho_{\bar{j}} \bar{R} u^{\alpha}}{|\partial \rho|_{\rho}^{2}-\rho}\right\} \\
= & \left(\frac{\rho}{r}\right) r_{\alpha \bar{\alpha}}\left\{X_{j} u^{\alpha} \overline{Y_{j} u^{\alpha}}+X_{j} \bar{u}^{\alpha} \bar{Y}_{j} u^{\alpha}+X_{j} u^{\alpha} \frac{\rho_{\bar{j}} \bar{R} \bar{u}^{\alpha}}{|\partial \rho|_{\rho}^{2}-\rho}+X_{j} \bar{u}^{\alpha} \frac{\rho_{\bar{j}} \bar{R} u^{\alpha}}{|\partial \rho|_{\rho}^{2}-\rho}\right\} \\
& +\left(\frac{\rho}{r}\right)\left(\frac{r_{\alpha} r_{\bar{\beta}}}{-r}\right)\left\{X_{j} u^{\alpha} \overline{Y_{j} u^{\beta}}+X_{j} \bar{u}^{\beta} \bar{Y}_{j} u^{\alpha}+X_{j} u^{\alpha} \frac{\rho_{\bar{j}} \bar{R} \bar{u}^{\beta}}{|\partial \rho|_{\rho}^{2}-\rho}+X_{j} \bar{u}^{\beta} \frac{\rho_{j} \bar{R} u^{\alpha}}{|\partial \rho|_{\rho}^{2}-\rho}\right\} \\
= & \left(\frac{\rho}{r}\right) r_{\alpha \bar{\alpha}}\left\{\rho_{j \bar{j}} X_{j} u^{\alpha} \overline{X_{j} u^{\alpha}}+\rho_{j \bar{j}} X_{j} \bar{u}^{\alpha} \bar{X}_{j} u^{\alpha}+X_{j} u^{\alpha} \rho_{\bar{j}} \frac{\bar{R} \bar{u}^{\alpha}}{|\partial \rho|_{\rho}^{2}-\rho}+X_{j} \bar{u}^{\alpha} \rho_{\bar{j}} \frac{\bar{R} u^{\alpha}}{|\partial \rho|_{\rho}^{2}-\rho}\right\} \\
& +\left(\frac{\rho}{r}\right)\left(\frac{1}{-r}\right)\left\{\rho_{j \bar{j}} r_{\alpha} X_{j} u^{\alpha} r_{\bar{\beta}} \overline{X_{j} u^{\beta}}+\rho_{j \bar{j}} r_{\bar{\beta}} X_{j} \bar{u}^{\beta} r_{\alpha} \bar{X} u_{j} u^{\alpha}\right\} \\
& +\left(\frac{\rho}{r}\right)\left(\frac{r_{\alpha} r_{\bar{\beta}}}{-r}\right)\left\{X_{j} u^{\alpha} \rho_{\bar{j}} \frac{\bar{R} \bar{u}^{\beta}}{|\partial \rho|_{\rho}^{2}-\rho}+X_{j} \bar{u}^{\beta} \rho_{\bar{j}} \frac{\bar{R} u^{\alpha}}{|\partial \rho|_{\rho}^{2}-\rho}\right\} .
\end{aligned}
$$

Using the fact $X_{j} u^{\alpha} \rho_{\bar{j}}=\frac{-\rho R u^{\alpha}}{|\partial \rho|_{\rho}^{2}-\rho}$ and Lemma 3.2 , we obtain

$$
\begin{aligned}
e & {[u]\left(z_{0}\right) } \\
= & \left(\frac{\rho}{r}\right) r_{\alpha \bar{\alpha}}\left\{\rho_{j \bar{j}} X_{j} u^{\alpha} \overline{X_{j} u^{\alpha}}+\rho_{j \bar{j}} X_{j} \bar{u}^{\alpha} \bar{X}_{j} u^{\alpha}+X_{j} u^{\alpha} \rho_{\bar{j}} \frac{\bar{R} \bar{u}^{\alpha}}{|\partial \rho|_{\rho}^{2}-\rho}+X_{j} \bar{u}^{\alpha} \rho_{\bar{j}} \frac{\bar{R} u^{\alpha}}{|\partial \rho|_{\rho}^{2}-\rho}\right\} \\
& +\left(\frac{\rho}{r}\right)\left(\frac{r_{\alpha} r_{\bar{\beta}}}{-r}\right)\left\{X_{j} u^{\alpha} \rho_{\bar{j}} \frac{\bar{R} \bar{u}^{\beta}}{|\partial \rho|_{\rho}^{2}-\rho}+X_{j} \bar{u}^{\beta} \rho_{\bar{j}} \frac{\bar{R} u^{\alpha}}{|\partial \rho|_{\rho}^{2}-\rho}\right\} \\
= & \left(\frac{\rho}{r}\right) r_{\alpha \bar{\alpha}}\left\{\rho_{j \bar{j}}\left|X_{j} u^{\alpha}\right|^{2}+\rho_{j \bar{j}}\left|\bar{X}_{j} u^{\alpha}\right|^{2}+\frac{(-\rho) R u^{\alpha}}{|\partial \rho|_{\rho}^{2}-\rho} \frac{\bar{R} \bar{u}^{\alpha}}{|\partial \rho|_{\rho}^{2}-\rho}+\frac{(-\rho) R \bar{u}^{\alpha}}{|\partial \rho|_{\rho}^{2}-\rho} \frac{\bar{R} u^{\alpha}}{|\partial \rho|_{\rho}^{2}-\rho}\right\} \\
& +\left(\frac{\rho}{r}\right)\left(\frac{r_{\alpha} r_{\bar{\beta}}}{-r}\right)\left\{\frac{(-\rho) R u^{\alpha}}{\left(|\partial \rho|_{\rho}^{2}-\rho\right)} \frac{\bar{R} \bar{u}^{\beta}}{\left(|\partial \rho|_{\rho}^{2}-\rho\right)}+\frac{(-\rho) R \bar{u}^{\beta}}{\left(|\partial \rho|_{\rho}^{2}-\rho\right)} \frac{\bar{R} u^{\alpha}}{\left(|\partial \rho|_{\rho}^{2}-\rho\right)}\right\} \\
= & \left(\frac{\rho}{r}\right) r_{\alpha \bar{\alpha}}\left\{\rho_{j \bar{j}}\left|X_{j} u^{\alpha}\right|^{2}+\rho_{j \bar{j}}\left|\bar{X}_{j} u^{\alpha}\right|^{2}\right\}
\end{aligned}
$$




$$
+\left(\frac{\rho}{r}\right) \frac{(-\rho)}{(-r)}\left\{\frac{r^{\alpha} R u^{\alpha}}{\left(|\partial \rho|_{\rho}^{2}-\rho\right)} \frac{r^{\bar{\beta}} \bar{R} \bar{u}^{\beta}}{\left(|\partial \rho|_{\rho}^{2}-\rho\right)}+\frac{r^{\alpha} R \bar{u}^{\alpha}}{\left(|\partial \rho|_{\rho}^{2}-\rho\right)} \frac{r^{\bar{\beta}} \bar{R} u^{\beta}}{\left(|\partial \rho|_{\rho}^{2}-\rho\right)}\right\} .
$$

Adding (3.22) to (3.23) and using Lemma 3.5, we get

$$
\begin{aligned}
& r_{\alpha \bar{\alpha}} \rho_{j \bar{j}}\left(\left|X_{j} u^{\alpha}\right|^{2}+\left|\bar{X}_{j} u^{\alpha}\right|^{2}\right) \\
& =\mathcal{L}(\rho) \frac{r_{s}(u)(R+\bar{R}) u^{s}}{|\partial \rho|_{\rho}^{2}}-2\left(r_{\alpha} \mathcal{L} u^{\alpha}+r_{\alpha \beta} X_{j} u^{\beta} \bar{X}_{j} u^{\alpha} \rho_{j \bar{j}}\right) \\
& =\mathcal{L}(\rho) a[u](z)-2\left(r_{\alpha} \mathcal{L} u^{\alpha}+r_{\alpha \beta} X_{j} u^{\beta} \bar{X}_{j} u^{\alpha} \rho_{j \bar{j}}\right) .
\end{aligned}
$$

We know from (3.42) that $\mathcal{L}(\rho)=m-1$ on $\partial \Omega_{m}$. Thus,

$$
\begin{aligned}
e[u]\left(z_{0}\right)= & \left(\frac{\rho}{r}\right)\left\{\mathcal{L}(\rho) a[u](z)-2\left(r_{\alpha} \mathcal{L} u^{\alpha}+r_{\alpha \beta} X_{j} u^{\beta} \bar{X}_{j} u^{\alpha} \rho_{j \bar{j}}\right)\right\} \\
& +\left(\frac{\rho}{r}\right)^{2}\left\{\frac{r^{\alpha} R u^{\alpha}}{\left(|\partial \rho|_{\rho}^{2}-\rho\right)} \frac{r^{\bar{\beta}} \bar{R} \bar{u}^{\beta}}{\left(|\partial \rho|_{\rho}^{2}-\rho\right)}+\frac{r^{\alpha} R \bar{u}^{\alpha}}{\left(|\partial \rho|_{\rho}^{2}-\rho\right)} \frac{r^{\bar{\beta}} \bar{R} u^{\beta}}{\left(|\partial \rho|_{\rho}^{2}-\rho\right)}\right\} \\
= & \left(\frac{\rho}{r}\right) \mathcal{L}(\rho) a[u](z)-2\left(\frac{\rho}{r}\right)\left(r_{\alpha} \mathcal{L} u^{\alpha}+r_{\alpha \beta} X_{j} u^{\beta} \bar{X}_{j} u^{\alpha} \rho_{j \bar{j}}\right) \\
& +\left(\frac{\rho}{r}\right)^{2}\left\{\frac{\left|r_{\alpha} R u^{\alpha}\right|^{2}}{\left(|\partial \rho|_{\rho}^{2}-\rho\right)^{2}}+\frac{\left|r_{\alpha} \bar{R} u^{\alpha}\right|^{2}}{\left(|\partial \rho|_{\rho}^{2}-\rho\right)^{2}}\right\} \\
= & \mathcal{L}(\rho)-2\left(\frac{\rho}{r}\right)\left(r_{\alpha} \mathcal{L} u^{\alpha}+r_{\alpha \beta} X_{j} u^{\beta} \bar{X}_{j} u^{\alpha} \rho_{j \bar{j}}\right) \\
& +\frac{\left|r_{\alpha} R u^{\alpha}\right|^{2}+\left|r_{\alpha} \bar{R} u^{\alpha}\right|^{2}}{(a[u](z))^{2}\left(|\partial \rho|_{\rho}^{4}\right)} .
\end{aligned}
$$

Lemma 3.5 tells us that $\left(r_{\alpha} R u^{\alpha}\right)$ and $\left(r_{\alpha} \bar{R} u^{\alpha}\right)$ are real. Thus,

$$
\begin{aligned}
\frac{\left|r_{\alpha} R u^{\alpha}\right|^{2}+\left|r_{\alpha} \bar{R} u^{\alpha}\right|^{2}}{(a[u](z))^{2}\left(|\partial \rho|_{\rho}^{4}\right)} & =\left(\frac{r_{\alpha}(R+\bar{R}) u^{\alpha}}{(a[u](z))\left(|\partial \rho|_{\rho}^{2}\right)}\right)^{2}-2 \frac{\left(r_{\alpha} R u^{\alpha}\right)\left(r_{\beta} \bar{R} u^{\beta}\right)}{(a[u](z))^{2}\left(|\partial \rho|_{\rho}^{4}\right)} \\
& =1-2 \frac{\left(r_{\alpha} R u^{\alpha}\right)\left(r_{\beta} \bar{R} u^{\beta}\right)}{(a[u](z))^{2}\left(|\partial \rho|_{\rho}^{4}\right)}
\end{aligned}
$$

by (3.32). As a result, we obtain

$$
\begin{aligned}
e[u]\left(z_{0}\right)= & (m-1)-2 \frac{\left(r_{\alpha} \mathcal{L} u^{\alpha}+r_{\alpha \beta} X_{j} u^{\beta} \bar{X}_{j} u^{\alpha} \rho_{j \bar{j}}\right)}{a[u]} \\
& +1-2 \frac{\left(r_{\alpha} R u^{\alpha}\right)\left(r_{\beta} \bar{R} u^{\beta}\right)}{(a[u](z))^{2}\left(|\partial \rho|_{\rho}^{4}\right)} \\
= & m-2 \frac{\left(r_{\alpha} \mathcal{L} u^{\alpha}+r_{\alpha \beta} X_{j} u^{\beta} \bar{X}_{j} u^{\alpha} \rho_{j \bar{j}}\right)}{a[u]}-2 \frac{\left(r_{\alpha} R u^{\alpha}\right)\left(r_{\beta} \bar{R} u^{\beta}\right)}{(a[u](z))^{2}\left(|\partial \rho|_{\rho}^{4}\right)} \\
= & m-2 \frac{\left(r_{\alpha} \mathcal{L} u^{\alpha}+r_{\alpha \beta} X_{j} u^{\beta} \bar{Y}_{j} u^{\alpha}\right)}{a[u]}-2 \frac{\left(r_{\alpha} R u^{\alpha}\right)\left(r_{\beta} \bar{R} u^{\beta}\right)}{(a[u](z))^{2}\left(|\partial \rho|_{\rho}^{4}\right)} \\
= & m-2 \frac{\left(r_{\alpha} \mathcal{L} u^{\alpha}+r_{\alpha \beta} X_{j} u^{\beta} u_{\bar{j}}^{\alpha}\right)}{a[u]}-2 \frac{\left(r_{\alpha} R u^{\alpha}\right)\left(r_{\beta} \bar{R} u^{\beta}\right)}{(a[u](z))^{2}\left(|\partial \rho|_{\rho}^{4}\right)} \\
= & m+2 \frac{\left(r_{\alpha} R u^{\alpha}\right)\left(r_{\beta} \bar{R} u^{\beta}\right)}{(a[u](z))^{2}\left(|\partial \rho|_{\rho}^{4}\right)}
\end{aligned}
$$

where the third equality comes from (3.30), the fourth from (3.28) and the last one from applying (3.26). The proof is complete. 
5. The proof of Theorem 1.1. In this section we prove Theorem 1.1. It is obvious that (i) implies (ii). Next, we prove that (ii) implies (iii). Without loss of generality we may assume that $\rho_{i \bar{j}}\left(z_{0}\right)=\delta_{i j} \rho_{i \bar{i}}\left(z_{0}\right)$. Since $u$ is pluriharmonic

$$
\begin{aligned}
0 & =\partial_{i \bar{j}} u^{s}+\sum_{t, \gamma=1}^{n} \Gamma_{t \gamma}^{s} \partial_{i} u^{t} \partial_{\bar{j}} u^{\gamma} \\
& =\partial_{i \bar{j}} u^{s}+\sum_{t, \gamma=1}^{n}\left[\frac{\delta_{t s} r_{\gamma}+r_{t} \delta_{\gamma s}}{-r}+\left(r^{s \bar{\ell}}-\frac{r^{s} r^{\bar{\ell}}}{|\partial r|_{r}^{2}-r}\right) r_{t \gamma \bar{\ell}}+\frac{r_{t \gamma} r^{s}}{\left.|\partial r|_{r}^{2}-r\right)}\right] \partial_{i} u^{t} \partial_{\bar{j}} u^{\gamma} .
\end{aligned}
$$

Multiplying by $(-\rho)$, we obtain that on $\partial \Omega_{m}$

$$
\begin{aligned}
0 & =\frac{1}{a[u]} \sum_{t, \gamma=1}^{n}\left(\delta_{t s} r_{\gamma}+r_{t} \delta_{\gamma s}\right) \partial_{i} u^{t} \partial_{\bar{j}} u^{\gamma} \\
& =\frac{1}{a[u]}\left(\sum_{\gamma=1}^{n} r_{\gamma} \partial_{i} u^{s} \partial_{\bar{j}} u^{\gamma}+\sum_{t=1}^{n} r_{t} \partial_{i} u^{t} \partial_{\bar{j}} u^{s}\right) \\
& =\frac{1}{a[u]}\left(\sum_{\gamma=1}^{n} r_{\gamma} \partial_{i} u^{s} \partial_{\bar{j}} u^{\gamma}+r_{\gamma} \partial_{i} u^{\gamma} \partial_{\bar{j}} u^{s}\right) .
\end{aligned}
$$

Multiplying by $r_{s}$ and adding over $s$ we have

$$
\begin{aligned}
0 & =\frac{1}{a[u]}\left(\sum_{\gamma, s=1}^{n} r_{s} \partial_{i} u^{s} r_{\gamma} \partial_{\bar{j}} u^{\gamma}+r_{\gamma} \partial_{i} u^{\gamma} r_{s} \partial_{\bar{j}} u^{s}\right) \\
& =\frac{2}{a[u]}\left(\sum_{\gamma, s=1}^{n} r_{s} \partial_{i} u^{s} r_{\gamma} \partial_{\bar{j}} u^{\gamma}\right) \\
& =\frac{2}{a[u]}\left(\sum_{\gamma, s=1}^{n} r_{s} \rho_{i \bar{i}}\left(X_{i} u^{s}+\frac{\rho^{\bar{i}}}{|\partial \rho|_{\rho}^{2}-\rho} R u^{s}\right) r_{\gamma} \rho_{i \bar{i}}\left(\bar{X}_{i} u^{\gamma}+\frac{\rho^{i}}{|\partial \rho|_{\rho}^{2}-\rho} \bar{R} u^{\gamma}\right)\right) \\
& =\frac{2}{a[u]}\left(\sum_{\gamma, s=1}^{n} r_{s} \rho_{i \bar{i}}\left(\frac{\rho^{\bar{i}}}{|\partial \rho|_{\rho}^{2}-\rho} R u^{s}\right) r_{\gamma} \rho_{i \bar{i}}\left(\frac{\rho^{i}}{|\partial \rho|_{\rho}^{2}-\rho} \bar{R} u^{\gamma}\right)\right) \\
& =\frac{2}{a[u]}\left(\sum_{\gamma, s=1}^{n} r_{s}\left(\frac{\rho_{i}}{|\partial \rho|_{\rho}^{2}-\rho} R u^{s}\right) r_{\gamma}\left(\frac{\rho_{\bar{i}}}{|\partial \rho|_{\rho}^{2}-\rho} \bar{R} u^{\gamma}\right)\right) \\
& =\frac{2}{a[u]}\left(\sum_{\gamma, s=1}^{n}\left|\rho_{i}\right|^{2}\left(\frac{r_{s} R u^{s}}{|\partial \rho|_{\rho}^{2}-\rho}\right)\left(\frac{r_{\gamma} \bar{R} u^{\gamma}}{|\partial \rho|_{\rho}^{2}-\rho}\right)\right)
\end{aligned}
$$

Thus, we have obtained that $\frac{2}{a[u]}\left(\sum_{\gamma, s=1}^{n}\left(\frac{r_{s} R u^{s}}{|\partial \rho|_{\rho}^{2}-\rho}\right)\left(\frac{r_{\gamma} \bar{R} u^{\gamma}}{|\partial \rho|_{\rho}^{2}-\rho}\right)\right)=0$ on $\partial \Omega_{m}$. It follows from (3.26) that $r_{s} \mathcal{L} u^{s}+r_{s t} X_{j} u^{t} u_{j}^{s}=0$ on $\partial \Omega_{m}$, which is (iii).

Next we show that (iii) implies (i). By assumption, $u$ is harmonic and $r_{s} \mathcal{L} u^{s}+$ $r_{s t} X_{j} u^{t} u \frac{s}{j}=0$ on $\partial \Omega_{m}$. By Theorem 3.4, we find that either $u$ or $\bar{u}$ is CR. Thus, there exists a holomorphic or antiholomorphic map $v$ such that $\left.v\right|_{\partial \Omega_{m}}=\left.u\right|_{\partial \Omega_{m}}$. Since $r$ is plurisubharmonic and $v$ is holomorphic or antiholomorphic, $r(v(z))$ is plurisubharmonic. Thus, by Hopf's lemma $a[v](p)=D_{\nu} r(v(p))>0$ at every $p \in \partial \Omega_{m}$. Thus by (iv) of Lemma 3.5 we obtain that $u \equiv v$ on $\Omega_{m}$. 
Now we proceed to show that (iii) implies (iv). Lemma 3.5 tells us that $E_{b}[u]>0$ on $\partial \Omega_{m}$ if and only if $a[u]>0$ on $\partial \Omega_{m}$. If $r_{s} \mathcal{L} u^{s}+r_{s t} X_{j} u^{t} u \frac{s}{j}=0$ on $\partial \Omega_{m}$, by (3.26) we obtain that $\left(r_{s} R u^{s}\right)\left(r_{t} \bar{R} u^{t}\right)=0$, which implies by Lemma 4.1 that $e[u](z)=m$ when $E_{b}[u]>0$.

Next, we show that (iv) implies (v). Lemma 3.5 tells us that $E_{b}[u]>0$ on $\partial \Omega_{m}$ if and only if $a[u]>0$ on $\partial \Omega_{m}$. Thus by (4.1) we have that $\left(r_{s} R u^{s}\right)\left(r_{t} \bar{R} u^{t}\right)=0$ on the set $\left\{z \in \partial \Omega_{m}: a[u](z)>0\right\}$. On the other hand, by (3.26) we obtain that $\left(r_{s} R u^{s}\right)\left(r_{t} \bar{R} u^{t}\right)=0$ on the set $\left\{z \in \partial \Omega_{m}: a[u](z)=0\right\}$. Thus (iv) implies (v). Finally, we show that (v) implies (iii). Using the hypothesis of (v) together with (3.26) and (3.32) we obtain that $r_{s} \mathcal{L} u^{s}+r_{s t} X_{j} u^{t} u \frac{s}{j}=0$ on $\partial \Omega_{m}$. Since (iii) implies (i) we are done.

6. The proof of Theorem 1.2. In this section we prove Theorem 1.2. Let $\phi \in C^{k, \alpha}\left(\partial \Omega_{m}\right)$ with $k \geq 2$ and $\alpha \geq 0$. Let $\phi(z)$ denote the 'radial' extension of $\phi$ from $\partial \Omega_{m}$ to $\bar{\Omega}_{m}$ in the sense that $r(\phi(z))=0$ for all $z \in \Omega_{m}$ near $\partial \Omega_{m}$. In order to apply Li-Tam's general existence theorem of [21], we first construct an approximating harmonic map similar to the construction in [25]. To do this, we define an extension $v(z)$ given by

$$
v(z)=\phi(z)+\rho(z) b(z),
$$

where $\rho(z)$ is a strictly plurisubharmonic defining function for $\Omega_{m}$, which is the potential function for the metric $h$, and $b(z)$ is a vector valued function which will be given later. A computation shows that

$$
\mathcal{L} v(z)=\mathcal{L} \phi(z)+\mathcal{L} \rho(z) b(z)+\rho(z) \mathcal{L} b+X_{i} \rho \partial_{\bar{i}} b+\bar{X}_{j} \rho \partial_{j} b(z),
$$

which implies that on $\partial \Omega_{m}$

$$
\mathcal{L} v(z)=\mathcal{L} \phi(z)+(m-1) b(z) .
$$

By (1.7) and (3.25), we have

$$
r_{s}(v) \tau^{s}[v]=-\rho(z)\left[r_{s}(v) \mathcal{L} v^{s}+r_{s t} X_{j} v^{t} v \frac{s}{j}+\frac{2 r_{s}(v) R v^{s} r_{t}(v) \bar{R} v^{t}}{a[v]\left(|\partial \rho|_{\rho}^{2}-\rho\right)^{2}}\right]+O\left(\rho^{2}\right) .
$$

Let

$$
I[v]=a[v]\left(r_{s}(v) \mathcal{L} v^{s}+r_{s t} X_{j} v^{t} v \frac{s}{j}\right)+\frac{2}{\left(|\partial \rho|_{\rho}^{2}-\rho\right)^{2}} r_{s}(v) R v^{s} r_{t}(v) \bar{R} v^{t} .
$$

Since $\sum_{j} \rho_{\bar{j}} X_{j}=O(\rho) R$, we have

$$
\sum_{j=1}^{n} X_{j} \phi^{t} v_{j}^{s}=\sum_{j=1}^{n} X_{j} \phi^{t} \overline{Y_{j}} v_{\bar{j}}^{s}+O(\rho)=\sum_{j=1}^{n} X_{j} \phi^{t} \overline{Y_{j}} \phi^{s}+O(\rho) .
$$

Let $b_{0} \geq 0$ and

$$
b^{s}=b_{0} r_{\bar{s}}(\phi), \quad 1 \leq s \leq n .
$$

Note that on $\partial \Omega_{m}$,

$$
a[v](z)=\frac{r(v(z))}{\rho(z)}=\frac{r(\phi+b \rho)}{\rho}=r_{s}(\phi+b \rho) b^{s}+r_{\bar{s}}(\phi+b \rho) \overline{b^{s}}=2 b_{0} \sum_{s}\left|r_{s}(\phi)\right|^{2} .
$$


Since we extended $\phi$ from $\partial \Omega_{m}$ to $\bar{\Omega}_{m}$ so that

$$
(R+\bar{R}) \phi=0, \quad \text { for } z \text { near } \partial \Omega_{m},
$$

and $r(\phi)=0$ on $\partial \Omega_{m}$, we have that

$$
2 \operatorname{Im} \sum_{s} r_{s}(\phi)(\bar{R}-R) \phi^{s}=0 .
$$

So $\sum_{s} r_{s}(\phi) \bar{R} \phi^{s}$ is real. By Lemma 3.3, we know that

$$
\sum_{j=1}^{m}\left(X_{j} \bar{Y}_{j}-\bar{X}_{j} Y_{j}\right)=\frac{m-1}{|\partial \rho|_{\rho}^{2}-\rho}(\bar{R}-R)+O(\rho)
$$

and

$$
\mathcal{L}=\frac{1}{2}\left(X_{j} \bar{Y}_{j}+\bar{X}_{j} Y_{j}\right)+\frac{m-1}{|\partial \rho|_{\rho}^{2}} \frac{(R+\bar{R})}{2}+O(\rho) .
$$

Since $r(\phi)=0$ on $\partial \Omega_{m}$ and $X_{j}$ is tangential we have that

$$
X_{j} r(\phi)=r_{s} X_{j} \phi^{s}+r_{\bar{s}} X_{j} \bar{\phi}^{s}=0 \text { on } \partial \Omega_{m} .
$$

Equation (6.5) together with (1.7) implies that

$$
\sum_{s} r_{s}(\phi) \bar{Y}_{j} \phi^{s}=\sum_{s} r_{s}(\phi) X_{j} \phi^{s}=0 \quad \text { on } \quad \partial \Omega_{m}
$$

As a result, we obtain that

$$
0=\sum_{s}\left(r_{s t} X_{j} \phi^{t}+r_{s \bar{t}}(\phi) X_{j} \overline{\phi^{t}}\right) \bar{Y}_{j} \phi^{s}+\sum_{s} r_{s}(\phi) X_{j} \bar{Y}_{j} \phi^{s}
$$

and

$$
0=\sum_{s}\left(r_{s t} \bar{X}_{j} \phi^{t}+r_{s \bar{t}}(\phi) \bar{X}_{j} \overline{\phi^{t}}\right) Y_{j} \phi^{s}+\sum_{s} r_{s}(\phi) \bar{X}_{j} Y_{j} \phi^{s} .
$$

Thus on $\partial \Omega_{m}$

$$
\begin{aligned}
& r_{s} \mathcal{L} v^{s}+r_{s t} X_{j} v^{s} v \frac{t}{j} \\
&=\frac{r_{s}}{2}\left(X_{j} \bar{Y}_{j}+\bar{X}_{j} Y_{j}\right) \phi^{s}+(m-1) r_{s} b^{s}+r_{s t} X_{j} v^{s} v \frac{t}{j} \\
&=\left(\frac{1}{2}\right)\left(r_{s} X_{j} \bar{Y}_{j} \phi^{s}+r_{s} \bar{X}_{j} Y_{j} \phi^{s}\right)+(m-1) r_{s} b^{s}+r_{s t} X_{j} v^{s} v \frac{t}{j} \\
&=\left(-\frac{1}{2}\right)\left(r_{s t}\left(X_{j} \phi^{t}\right)\left(\bar{Y}_{j} \phi^{s}\right)+r_{s \bar{t}}\left(X_{j} \bar{\phi}^{t}\right)\left(\bar{Y}_{j} \phi^{s}\right)+r_{s t}\left(\bar{X}_{j} \phi^{t}\right)\left(Y_{j} \phi^{s}\right)\right. \\
&\left.+r_{s \bar{t}}\left(\bar{X}_{j} \bar{\phi}^{t}\right)\left(Y_{j} \phi^{s}\right)\right)+(m-1) r_{s} b^{s}+r_{s t} X_{j} v^{s} v \frac{t}{j} \\
&=-\left(\frac{1}{2}\right)\left(r_{s t}\left(X_{j} \phi^{t}\right)\left(\bar{Y}_{j} \phi^{s}\right)+r_{s t}\left(\bar{X}_{j} \phi^{t}\right)\left(Y_{j} \phi^{s}\right)+r_{s \bar{t}}\left(X_{j} \bar{\phi}^{t}\right)\left(\bar{Y}_{j} \phi^{s}\right)\right. \\
&\left.+r_{s \bar{t}}\left(\bar{X}_{j} \bar{\phi}^{t}\right)\left(Y_{j} \phi^{s}\right)\right)+(m-1) r_{s} b^{s}+r_{s t} X_{j} v^{s} v_{\bar{j}}^{t} .
\end{aligned}
$$


It is easy to see that on $\partial \Omega_{m}$

$$
r_{s} R v^{s}=r_{s}\left(R \phi^{s}+R(\rho) b^{s}\right)=r_{s} R \phi^{s}+|\partial \rho|_{\rho}^{2} r_{s} b^{s}=r_{s} R \phi^{s}+|\partial \rho|_{\rho}^{2} \frac{a[v]}{2}
$$

and

$$
r_{s} \bar{R} v^{s}=r_{s} \bar{R} \phi^{s}+|\partial \rho|_{\rho}^{2} \frac{a[v]}{2} .
$$

Let $z_{0} \in \partial \Omega_{m}$. By a rotation if necessary, we may assume without loss of generality that $\rho_{i \bar{j}}\left(z_{0}\right)=\rho_{i \bar{i}}\left(z_{0}\right) \delta_{i j}$. Then at $z_{0}$

$$
Y_{j} \phi^{s}=\rho_{j \bar{j}} X_{j} \phi^{s} \text { and } \bar{Y}_{j} \phi^{s}=\rho_{j \bar{j}} \bar{X}_{j} \phi^{s} .
$$

Also

$$
\begin{aligned}
r_{s t}(v) X_{j} v^{t} v \frac{s}{j}\left(z_{0}\right) & =r_{s t}(v) X_{j} v^{t} \bar{Y}_{j} v^{s}\left(z_{0}\right)+r_{s t}(v) X_{j} v^{t} \rho_{\bar{j}} \rho_{k} \partial_{\bar{k}} v^{s}\left(z_{0}\right) \\
& =r_{s t}(v) X_{j} v^{t} \bar{Y}_{j} v^{s}\left(z_{0}\right) \\
& =r_{s t}(v) X_{j} v^{t} \bar{Y}_{j} \phi^{s}\left(z_{0}\right) \\
& =r_{s t}(\phi)\left(X_{j} \phi^{t}\right)\left(\rho_{j j} \bar{X}_{j} \phi^{s}\right)\left(z_{0}\right) .
\end{aligned}
$$

Therefore at $z_{0}$,

$$
r_{s} \mathcal{L} v^{s}+r_{s t} X_{j} v^{s} v \frac{t}{j}=-\frac{1}{2} r_{s \bar{t}}\left(\bar{X}_{j} \overline{\phi^{t}} Y_{j} \phi^{s}+X_{j} \bar{\phi}^{t} \bar{Y}_{j} \phi^{s}\right)+(m-1) r_{s}(\phi) b^{s}
$$

and by (6.6) and (6.7)

$$
\sum_{j=1}^{m} r_{s}\left(X_{j} \bar{Y}_{j}-\bar{X}_{j} Y_{j}\right) \phi^{s}=-r_{s \bar{t}}\left(X_{j} \bar{\phi}^{t}\right)\left(\bar{Y}_{j} \phi^{s}\right)+r_{s \bar{t}}\left(\bar{X}_{j} \bar{\phi}^{t}\right)\left(Y_{j} \phi^{s}\right) .
$$

Thus, we obtain

$$
\begin{aligned}
I[v]= & a[v]\left(r_{s} \mathcal{L} v^{s}+r_{s t} X_{j} v^{s} v \frac{t}{j}\right)+2 \frac{\left(r_{s} R v^{s}\right)\left(r_{t} \bar{R} v^{t}\right)}{|d \rho|^{4}} \\
= & a[v]\left(\frac{r_{s \bar{t}}\left(X_{j} \bar{\phi}^{t}\right)\left(\bar{Y}_{j} \phi^{s}\right)+r_{s \bar{t}}\left(\bar{X}_{j} \bar{\phi}^{t}\right)\left(Y_{j} \phi^{s}\right)}{-2}+(m-1) r_{s} b^{s}\right) \\
& +\frac{1}{2}\left(\frac{|d \rho|^{4} a[v]^{2}-\left(r_{s}(R-\bar{R}) \phi^{s}\right)^{2}}{|d \rho|^{4}}\right) \\
= & 2\left|r_{s}\right|^{2} b_{0}\left(\frac{r_{s \bar{t}}\left(X_{j} \bar{\phi}^{t}\right)\left(\bar{Y}_{j} \phi^{s}\right)+r_{s \bar{t}}\left(\bar{X}_{j} \bar{\phi}^{t}\right)\left(Y_{j} \phi^{s}\right)}{-2}+(m-1)\left|r_{s}\right|^{2} b_{0}\right) \\
& +\frac{1}{2}\left(\left(2\left|r_{s}\right|^{2} b_{0}\right)^{2}-\frac{\left(r_{s}\left(X_{j} \bar{Y}_{j}-\bar{X}_{j} Y_{j}\right) \phi^{s}\right)^{2}}{(m-1)^{2}}\right) \\
= & 2\left|r_{s}\right|^{2} b_{0}\left(\frac{r_{s \bar{t}}\left(X_{j} \bar{\phi}^{t}\right)\left(\bar{Y}_{j} \phi^{s}\right)+r_{s \bar{t}}\left(\bar{X}_{j} \bar{\phi}^{t}\right)\left(Y_{j} \phi^{s}\right)}{-2}+(m-1)\left|r_{s}\right|^{2} b_{0}\right) \\
& +\frac{1}{2}\left(\left(2\left|r_{s}\right|^{2} b_{0}\right)^{2}-\frac{-\left(r_{s \bar{t}}\left(X_{j} \bar{\phi}^{t}\right)\left(\bar{Y}_{j} \phi^{s}\right)+r_{s \bar{t}}\left(\bar{X}_{j} \bar{\phi}^{t}\right)\left(Y_{j} \phi^{s}\right)\right)^{2}}{(m-1)^{2}}\right) .
\end{aligned}
$$


Let

$$
\begin{aligned}
& A=r_{s \bar{t}}\left(X_{j} \bar{\phi}^{t}\right)\left(\bar{Y}_{j} \phi^{s}\right), \\
& B=r_{s \bar{t}}\left(\bar{X}_{j} \bar{\phi}^{t}\right)\left(Y_{j} \phi^{s}\right), \\
& E=A+B .
\end{aligned}
$$

Then

$$
\begin{aligned}
I[v] & =2\left|r_{s}\right|^{2} b_{0}\left(\frac{-E}{2}+(m-1)\left|r_{s}\right|^{2} b_{0}\right)+2\left|r_{s}\right|^{4} b_{0}^{2}-\frac{1}{2(m-1)^{2}}(-A+B)^{2} \\
& =2 m\left|r_{s}\right|^{4} b_{0}^{2}-E\left|r_{s}\right|^{2} b_{0}-\frac{(-A+B)^{2}}{2(m-1)^{2}} .
\end{aligned}
$$

Let

$$
\begin{aligned}
4 m\left|r_{s}\right|^{2} b_{0} & =E+\sqrt{E^{2}+\frac{4 m}{(m-1)^{2}}(-A+B)^{2}} \\
& =E+\frac{1}{m-1} \sqrt{(m-1)^{2} E^{2}+4 m\left(E^{2}-4 A B\right)} \\
& =E+\frac{1}{m-1} \sqrt{(m+1)^{2} E^{2}-16 m A B} \\
& \geq E+\frac{1}{m-1} \sqrt{(m+1)^{2} E^{2}-4 m E^{2}} \\
& =2 E .
\end{aligned}
$$

By assumption $E_{b}[\phi]>0$ on $\partial \Omega_{m}$, which implies that $E>0$ since $r_{s \bar{t}}$ is positive definite. This in turn implies that $b_{0}>0$ and $a[v]=2 b_{0}>0$ on $\Omega_{m}$. Moreover,

$$
I[v]=a[v]\left(r_{s} \mathcal{L} v^{s}+r_{s t} X_{j} v^{s} v \frac{t}{j}\right)+2 \frac{\left(r_{s} R v^{s}\right)\left(r_{t} \bar{R} v^{t}\right)}{|\partial \rho|_{\rho}^{4}}=0 \text { on } \partial \Omega_{m} .
$$

Note that $\sum_{s} r_{s} \tau^{s}[v]=O\left(\rho^{2}\right)$ by (6.4). Thus,

$$
\frac{\left|\sum_{s} r_{s}(v) \tau^{s}[v]\right|^{2}}{r(v)^{2}}=O\left(\rho^{2}\right)
$$

By (3.24), we have

$$
\tau^{s}[v]=O(\rho)
$$

Therefore,

$$
\begin{aligned}
|\tau[v]|_{g}^{2} & =g_{\alpha \bar{\beta}} \tau^{\alpha}[v] \overline{\tau^{\beta}[v]} \\
& =\frac{\sum_{\alpha} r_{\alpha \bar{\beta}} \tau^{\alpha}[v] \overline{\tau^{\beta}[v]}}{|r(v)|}+\frac{r_{\alpha} \tau^{\alpha}[v] \overline{r_{\beta} \tau^{\beta}[v]}}{r(v)^{2}} \\
& =O(\rho(z)) .
\end{aligned}
$$

This implies $|\tau[v]|_{g} \in L^{2 p}\left(\Omega_{m}, d \lambda_{m}\right)$ for $p>m$ where $d \lambda_{m}(z)=\operatorname{det}\left(h_{i \bar{j}}\right) d v(z)$ and $\operatorname{det}\left(h_{i \bar{j}}\right)(z) \approx|\rho(z)|^{-m-1} d v(z)$ for all $z \in \Omega_{m}$ since $\Omega_{m}$ is strictly pseudoconvex.

An application of the existence theorem of [19] and the regularity argument in [20] establishes our claim. The proof of Theorem 1.2 is complete. 


\section{REFERENCES}

[1] T. Aubin, Some Nonlinear Problems in Riemannian Geometry, Springer-Verlag, Berlin, Heidelberg and New York, 1998.

[2] S. M. Baouendi, P. Ebenfelt and L. P. Rothschild, Real Submanifolds in Complex Space and their Mappings, Math Series 47, Princeton University Press, Princeton, New Jersey, 1999.

[3] M. Beals, C. Fefferman and R. Grossman, Strictly pseudoconvex domains in $\mathbb{C}^{n}$, Bull. Amer. Math. Soc. (N.S.), 8:2 (1983), pp. 125-322.

[4] S. Bochner, Analytic and meromorphic continuation by means of Green's formula, Ann. of Math., 44 (1943), pp. 652-673.

[5] A. Boggess, CR Manifolds and the Tangential Cauchy-Riemann Complex, CRC Press, Boca Raton, FL, 1991.

[6] L. Caffarelli, J. J. Kohn, L. Nirenberg and J. Spruck, The Dirichlet problem for nonlinear second-order elliptic equations. II. Complex Monge-Ampère, and uniformly elliptic, equations, Comm. Pure Appl. Math., 38 (1985), pp. 209-252.

[7] J-Y. Chen And S-Y. Li, Holomorphic extensions of maps from the boundary of Kähler manifolds, Tohoku Math. J. (2), 49:4 (1997), pp. 585-597.

[8] S. Y. Cheng And S. T. YAU, On the existence of a complex Kähler metric on non-compact complex manifolds and the regularity of Fefferman's equation, Comm. Pure Appl. Math., 33 (1980), pp. 507-544.

[9] K. Diederich And J. E. Fornæss, Pseudoconvex domains: An example with nontrivial Nebenhülle, Math. Ann., 225 (1977), pp. 275-292.

[10] K. Diederich and T. Ohsawa, An estimate for the Bergman distance on pseudoconvex domains, Annals of Math., 141 (1995), pp. 181-190.

[11] H. Donnelly, Dirichlet problem at infinity for harmonic maps: Rank one symmetric spaces, Trans. Am. Math. Soc., 344 (1994), pp. 713-735.

[12] J. Eells and J. H. SAmpson, Harmonic mappings of Riemannian manifolds, Amer. J. Math., 86 (1964), pp. 109-160.

[13] J. Eells and L. Lemaire, A report on harmonic maps, Bull. Lond. Math. Soc., 10 (1978), pp. 1-68.

[14] J. Eells and L. Lemaine, Another report on harmonic maps, Bull. Lond. Math. Soc., 20 (1988), pp. 385-524.

[15] C. FefFerman, The Bergman kernel and biholomorphic mappings of pseudoconvex domains, Invent. Math., 31 (1979), pp. 131-262.

[16] C. R. Graham and J. M. LeE, Smooth solutions of degenerate Laplacians on strictly pseudoconvex domains, Duke Math. J., 57 (1988), pp. 697-720.

[17] P. KLEMBECK, Kähler metrics of negative curvature, the Bergman metric near the boundary and the Kobayashi metric on smooth bounded strictly pseudoconvex sets, Indiana Univ. Math. J., 27 (1978), pp. 275-282.

[18] S. G. Krantz and S.-Y. LI, On the Existence of Smooth Plurisubharmonic Solutions for Certain Degenerate Monge-Ampère Equations, Complex variables, 41 (2000), pp. 207-219.

[19] P. Li AND L. F. TAM, The heat equation and harmonic maps of complete manifolds, Invent. Math., 105 (1991), pp. 1-46.

[20] P. Li And L. F. TAM, Uniqueness and regularity of proper harmonic maps, Ann. of Math., 137 (1993), pp. 167-201.

[21] P. Li AND L. F. TAM, Uniqueness and regularity of proper harmonic maps II, Indiana Univ. Math. J., 42 (1993), pp. 591-635.

[22] S.-Y. LI, The Neumann Problem for Complex Monge-Ampère equation, Indiana University J. of Mathematics, 44 (1995), pp. 1099-1122.

[23] S.-Y. LI, On the Dirichlet Problem for Symmetric Function Equations of Eigenvalues of Complex Hessian, Asian J. Math., 8 (2004), pp. 87-106.

[24] S.-Y. LI, Characterization for Balls with Potential Function of Kähler-Einstein Metrics for domains in $\mathbb{C}^{n}$, Comm. in Analysis and Geometry, 13 (2005), pp. 461-478.

[25] S-Y. LI AND L. NI, On the holomorphicity of proper harmonic maps between unit balls with the Bergman metrics, Math. Ann., 316 (2000), pp. 333-354.

[26] S-Y. Li AND E. Simon, Boundary behavior of harmonic functions in metrics of Bergman type in the polydisc, American Journal of Mathematics, 124 (2002), pp. 1045-1057.

[27] S-Y. Li AND W. ZHANG, CR functions on boundary of a strictly pseudoconvex hypersurfaces, preprint.

[28] Lei Ni AND L-F. TAM, Plurisubharmonic functions and the structure of complete Kähler manifolds with nonnegative curvature, J. Differential Geom., 64:3 (2003), pp. 457-524. 
[29] J. H. SAmpson, Application of harmonic maps to Kähler geometry, Contemp. Math., 49 (1986), pp. $125-134$.

[30] R. SCHOEN AND S. T. YAU, Compact group actions and the topology of manifolds with nonpositive curvature, Topology, 18 (1979), pp. 361-380.

[31] Y.T. SiU, The complex-analyticity of harmonic maps and the strong rigidity of compact Kähler manifolds, Ann. of Math., 112 (1980), pp. 73-111.

[32] Y.T. Siu, Complex-analyticity of harmonic maps, vanishing and Lefschetz theorems, J. Diff. Geom., 17 (1982), pp. 55-138.

[33] J. WANG, The heat flow and harmonic maps between complete manifolds, J. Geom. Anal., 8:3 (1998), pp. 485-514. 
